\title{
What Does a Submillimeter Galaxy Selection Actually Select? The Dependence of Submillimeter Flux Density on Star Formation Rate and Dust Mass
}

\section{Citation}

Hayward, Christopher C., Dušan Kereš, Patrik Jonsson, Desika Narayanan, T. J. Cox, and Lars Hernquist. 2011. "WHAT DOES A SUBMILLIMETER GALAXY SELECTION ACTUALLY SELECT?

THE DEPENDENCE OF SUBMILLIMETER FLUX DENSITY ON STAR FORMATION RATE AND DUST MASS." The Astrophysical Journal 743 (2): 159. https://doi.org/10.1088/0004-637x/743/2/159.

\section{Permanent link}

http://nrs.harvard.edu/urn-3:HUL.InstRepos:41381843

\section{Terms of Use}

This article was downloaded from Harvard University's DASH repository, and is made available under the terms and conditions applicable to Open Access Policy Articles, as set forth at http:// nrs.harvard.edu/urn-3:HUL.InstRepos:dash.current.terms-of-use\#OAP

\section{Share Your Story}

The Harvard community has made this article openly available.

Please share how this access benefits you. Submit a story.

\section{Accessibility}


Draft version August 20, 2018

Preprint typeset using LATEX style emulateapj v. 5/25/10

\title{
WHAT DOES A SUBMILLIMETER GALAXY SELECTION ACTUALLY SELECT? THE DEPENDENCE OF SUBMILLIMETER FLUX DENSITY ON STAR FORMATION RATE AND DUST MASS
}

\author{
Christopher C. Hayward ${ }^{1,2}$, Dušan Kere $\check{\check{S}}^{1,3,4}$, Patrik Jonsson ${ }^{1}$, \\ Desika NARAYAnan ${ }^{1,5,6}$, T. J. Cox ${ }^{7}$, AND Lars HeRnquist ${ }^{1}$ \\ Draft version August 20, 2018
}

\begin{abstract}
We perform 3-D dust radiative transfer (RT) calculations on hydrodynamic simulations of isolated and merging disk galaxies in order to quantitatively study the dependence of observed-frame submillimeter (submm) flux density on galaxy properties. We find that submm flux density and star formation rate (SFR) are related in dramatically different ways for quiescently star-forming galaxies and starbursts. Because the stars formed in the merger-induced starburst do not dominate the bolometric luminosity and the rapid drop in dust mass and more compact geometry cause a sharp increase in dust temperature during the burst, starbursts are very inefficient at boosting submm flux density (e.g., a $\gtrsim 16 \mathrm{x}$ boost in SFR yields a $\lesssim 2 \mathrm{x}$ boost in submm flux density). Moreover, the ratio of submm flux density to SFR differs significantly between the two modes; thus one cannot assume that the galaxies with highest submm flux density are necessarily those with the highest bolometric luminosity or SFR. These results have important consequences for the bright submillimeter-selected galaxy (SMG) population. Among them are: 1. The SMG population is heterogeneous. In addition to merger-driven starbursts, there is a subpopulation of galaxy pairs, where two disks undergoing a major merger but not yet strongly interacting are blended into one submm source because of the large ( $\gtrsim 15 "$, or $\sim 130 \mathrm{kpc}$ at $z=2$ ) beam of single-dish submm telescopes. 2. SMGs must be very massive $\left(M_{\star} \gtrsim 6 \times 10^{10} M_{\odot}\right)$. 3. The infall phase makes the SMG duty cycle a factor of a few greater than what is expected for a merger-driven starburst. Finally, we provide fitting functions for SCUBA and AzTEC submm flux densities as a function of SFR and dust mass and bolometric luminosity and dust mass; these should be useful for calculating submm flux density in semi-analytic models and cosmological simulations when performing full RT is computationally not feasible.

Subject headings: galaxies: high-redshift — galaxies: interactions — galaxies: starburst — infrared: galaxies — radiative transfer — submillimeter: galaxies
\end{abstract}

\section{INTRODUCTION}

Submillimeter-selected galaxies (SMGs: Smail et al. 1997; Barger et al. 1998; Hughes et al. 1998; Eales et al. 1999; see Blain et al. 2002 for a review) are extremely luminous (bolometric luminosity $L_{\mathrm{bol}} \sim 10^{12}-10^{13} L_{\odot}$; e.g., Kovács et al. 2006), high-redshift (Chapman et al. 2005) galaxies powered primarily by star formation rather than AGN (Alexander et al. 2005a, b, 2008; Valiante et al. 2007; Menéndez-Delmestre et al. 2007, 2009; Pope et al. 2008; Younger et al. 2008, 2009b). Because of their high dust content, SMGs emit almost all of their luminosity in the IR. As the name suggests, a galaxy is defined as an SMG if it is detected in the submm (historically, $850 \mu \mathrm{m}$ flux density $S_{850} \gtrsim 3-5$ $\mathrm{mJy}$; the nature of the population is sensitive to the adopted flux density cut, so we define an SMG as a

\footnotetext{
${ }^{1}$ Harvard-Smithsonian Center for Astrophysics, 60 Garden Street, Cambridge, MA 02138, USA

2 chayward@cfa.harvard.edu

${ }^{3}$ Department of Astronomy and Theoretical Astrophysics Center, University of California Berkeley, Berkeley, CA 94720, USA

${ }^{4}$ Hubble Fellow

${ }^{5}$ Steward Observatory, Department of Astronomy, University of Arizona, 933 North Cherry Avenue, Tucson, AZ 85721, USA

${ }^{6}$ Bart J. Bok Fellow

${ }^{7}$ Carnegie Observatories, 813 Santa Barbara Street, Pasadena, CA 91101, USA
}

source with $S_{850}>3 \mathrm{mJy}$ ), which requires $L_{\mathrm{IR}} \gtrsim 10^{12} L_{\odot}$ (Kovács et al. 2006; Coppin et al. 2008), so SMGs are typically ultra-luminous infrared galaxies (ULIRGs). Locally, ULIRGs are almost exclusively merging galaxies (Sanders \& Mirabel 1996; Lonsdale et al. 2006), so one might expect that at least some SMGs are also merging galaxies. Indeed, many observations support a merger origin for SMGs (e.g., Ivison et al. 2002, 2007, 2010; Chapman et al. 2003; Neri et al. 2003; Smail et al. 2004; Swinbank et al. 2004; Greve et al. 2005; Tacconi et al. 2006, 2008; Bouché et al. 2007; Biggs \& Ivison 2008; Capak et al. 2008; Younger et al. 2008, 2010; Iono et al. 2009; Engel et al. 2010). Furthermore, in Narayanan et al. (2010a, hereafter N10) we combined hydrodynamic simulations and radiative transfer (RT) calculations to show that major mergers can reproduce the full range of submm flux densities and typical UV-mm spectral energy distribution (SED) of SMGs (cf. Chakrabarti et al. 2008; Chakrabarti \& Whitney 2009). Semi-analytic models also predict that the SMG population is dominated by merger-induced starbursts rather than quiescent star formation (Baugh et al. 2005; Fontanot et al. 2007; Swinbank et al. 2008; Lo Faro et al. 2009; Fontanot \& Monaco 2010; González et al. 2011; but cf. Granato et al. 2004).

However, because of the much greater rate of gas 
supply onto galaxies at high redshift (e.g., Kereš et al. 2005; Dekel et al. 2009), gas fractions (Erb et al. 2006; Tacconi et al. 2006, 2010; Daddi et al. 2010) and star formation rates (Daddi et al. 2007; Noeske et al. 2007a, b) of galaxies at fixed galaxy mass increase rapidly with redshift. Thus, at $z \sim 2-3$ even a "normal" starforming galaxy can reach ULIRG luminosities (e.g., Hopkins et al. 2008c, 2010; Daddi et al. 2005, 2007; Dannerbauer et al. 2009). Furthermore, roughly estimating submm counts using estimates of high-redshift major merger rates and the short duty cycle of mergerinduced starbursts suggests that there may not be enough major mergers to account for the SMG population (Davé et al. 2010). This motivates the view that, instead, typical SMGs may be massive, gas-rich disks quiescently forming stars and fueled by continuous gas supply from mergers and smooth accretion Carilli et al. 2010, but cf. Daddi et al. 2009b).

The mode of star formation responsible for the majority of the SMG population is still a matter of debate, as it is difficult to discriminate between the two scenarios given the currently available data. A better understanding of the submm galaxy selection can clarify the nature of the SMG population.

Since SMGs have redshifts $z \sim 1-4$ (Dannerbauer et al. 2002; Chapman et al. 2005. Younger et al. 2007, 2008; Capak et al. 2008; Greve et al. 2008; Schinnerer et al. 2008; Daddi et al. 2009a. b; Knudsen et al. 2010), the observed submm flux density traces rest-frame $\sim 150-400 \mu \mathrm{m}$, longward of the peak of the IR SED. Thus the observed submm flux density is sensitive to both the total IR luminosity and the "dust temperature" 8 of the SED, which depend on the luminosity from stars and AGN absorbed by the dust, the mass and composition of the dust, and the spatial distribution of stars, AGN, and dust. Galaxies do not have identical SED shapes, so the dependence on dust temperature implies that galaxies with the highest submm flux density are not necessarily those with the highest bolometric luminosity. Furthermore, because star formation histories are more complicated than an instantaneous burst, the luminosity and instantaneous SFR are not necessarily linearly proportional. Thus the relationship between submm flux density and SFR is potentially more complicated than the relationship between submm flux density and bolometric luminosity. We therefore cannot say a priori that the galaxies with the highest submm flux densities are the most rapidly star-forming or most luminous bolometrically. Indeed, it has already been observationally demonstrated that submm selection does not select all the brightest galaxies in a given volume, as there are galaxies with luminosities and redshifts comparable to those of SMGs that are undetected in the submm because of their relatively hot SEDs (Chapman et al. 2004; Chapman et al. 2010; Casev et al. 2009; Casev et al. 2010; Hwang et al. 2010; Magdis et al. 2010; Magnelli et al. 2010). A submm galaxy selection is clearly biased toward cold galaxies;

\footnotetext{
8 As is convention, we will use the term "dust temperature" to denote the temperature derived from a single-temperature modified blackbody fit to the SED. This is simply a parameterization of the SED shape rather than a physical temperature. In our simulations dust grains have a continuum of temperatures, depending on both grain size and the local radiation field heating the dust.
}

however, the details of the selection bias are yet to be understood.

Despite the basic physical reasons that one does not expect a simple relation between submm flux density and SFR, a linear relation between submm flux and SFR has been used explicitly (and, even more frequently, implicitly) to infer SFR from observed submm flux densities (e.g., Chapman et al. 2000; Peacock et al. 2000; Blain et al. 2002; Scott et al. 2002; Webb et al. 2003; van Kampen et al. 2005; Tacconi et al. 2008; Wang et al. 2011), typically because the data sets do not have enough photometric data points to precisely constrain the IR SED shape (Herschel data are already helping greatly in this regard; e.g., Chapman et al.|2010; Dannerbauer et al. 2010; Magnelli et al.|2010). Furthermore, some theoretical studies (Davé et al. 2010) have assumed that SMGs are the most rapidly star-forming galaxies in order to identify SMGs in cosmological simulations without performing RT. If SFR and submm flux density are not simply related this approach is problematic.

It is clear that a better understanding of the relationship between submm flux density and SFR and, more generally, what galaxy properties a submm galaxy selection selects for, is needed. In other work we have combined hydrodynamic simulations and dust RT to show that major mergers of massive, gas-rich disk galaxies can reproduce the $850 \mu \mathrm{m}$ flux densities (N10), CO properties (Naravanan et al. 2009), number densities (Hayward et al. 2011, C. Hayward et al. 2011, in preparation), and intersection with the dust-obscured galaxy (DOG) population (Narayanan et al. 2010b) of SMGs. Motivated by the success of our simulations in reproducing a variety of SMG properties, we use them here to quantify how submm flux density depends on SFR, $L_{\mathrm{bol}}$, dust content, and geometry. The aim of this study is to clarify for what galaxy properties a submm selection criterion selects and to provide a discriminant among the different modes of star formation that could power SMGs.

\section{METHODS}

We combine high-resolution GADGET2 (Springel et al. 2001; Springel 2005) 3-D Nbody/smoothed-particle hydrodynamics (SPH) simulations with the SUNRISE (Jonsson 2006; Jonsson et al. 2010) polychromatic Monte Carlo dust RT code in order to predict the submillimeter flux densities of high-redshift isolated and merging disk galaxies. The simulations presented here, part of a larger suite to be presented in C. Hayward et al. (2011, in preparation), are described in N10, so here we will only summarize and describe differences from that work. This combination of GADGET-2 and SUNRISE has been successfully shown to reproduce the SEDs/colors of local SINGS (Kennicutt et al. 2003; Dale et al. 2007) galaxies (Jonsson et al. 2010); local ULIRGs (Younger et al. 2009a); massive, quiescent, compact $z \sim 2$ galaxies (Wuyts et al. 2009, 2010); $24 \mu \mathrm{m}$-selected galaxies (Naravanan et al. 2010b); K+A/post-starburst galaxies (Snvder et al. 2011); and XUV disks (Bush et al. 2010), among other populations. The success of our approach at modeling diverse galaxy populations - both local and high-redshift-lends credibility to its application to 
modeling SMGs.

\subsection{Hydrodynamic simulations}

GADGET-29 (Springel et al. 2001; Springel 2005) is a TreeSPH (Hernquist \& Katz 1989) code that computes gravitational interactions via a hierarchical tree method (Barnes \& Hut 1986) and gas dynamics via SPH (Lucy 1977; Gingold \& Monaghan 1977). It conserves both energy and entropy (Springel \& Hernquist 2002). The simulations include radiative heating and cooling as in Katz et al. (1996). Star formation is modeled via the volume-density dependent Kennicutt-Schmidt law Kennicutt 1998a), $\rho_{\text {SFR }} \propto \rho_{\text {gas }}^{1.5}$, with a minimum density threshold; this index is consistent with observations of $z \sim 2$ disks (Krumholz \& Thompson 2007; Naravanan et al. 2008a, 2011). The density threshold used is $n \sim 0.1 \mathrm{~cm}^{-3}$, much less than that of the dense molecular gas from which stars form $\left(n \sim 10^{2}-\right.$ $\left.10^{3} \mathrm{~cm}^{-3}\right)$. For this reason, and because we do not track the formation of molecular gas, the KS law employed should be considered simply an empirically- and physically-motivated prescription to encapsulate physics we do not resolve. The SF prescription has been calibrated to reproduce the global K-S law (see $\S 3$ of Springel \& Hernquist 2003). Recently, some authors (e.g., Hopkins et al. 2011) have presented simulations that resolve the density threshold for molecular gas formation; we plan to compare such simulations to our current simulations in future work.

The structure of the ISM is modeled via a two-phase sub-resolution model in which cold, dense clouds are embedded in a diffuse, hot medium (Springel \& Hernquist 2003). This medium is pressurized by supernova feedback, which heats the diffuse ISM and evaporates the cold clouds (Cox et al. 2006b). Metal enrichment is calculated by assuming each gas particle behaves as a closed box. Black hole particles accrete via Eddington-limited Bondi-Hoyle accretion and deposit $5 \%$ of their emitted luminosity - calculated from the accretion rate assuming $10 \%$ radiative efficiency, $L_{\mathrm{bol}}=0.1 \dot{M} c^{2}$ - to the surrounding ISM as thermal energy (Springel et al. 2005; Matteo et al. 2005). We refer the reader to the references given above for the full details of the GADGET- 2 code and the sub-resolution models employed.

We focus on two simulations, one isolated disk and one major merger. We embed exponential disks with baryonic mass $4 \times 10^{11} M_{\odot}$ in $9 \times 10^{12} M_{\odot}$ dark matter halos described by a Hernquist (1990) density profile. The disks are initially $60 \%$ gas and are scaled to $z \sim 3$ as described in Robertson et al. (2006a b). The gravitational softening lengths are $200 h^{-1}$ pc for the dark matter particles and $100 h^{-1}$ pc for the star, gas, and black hole particles. We use $6 \times 10^{4}$ dark matter, $4 \times 10^{4}$ stellar, $4 \times 10^{4}$ gas, and 1 black hole particle per disk galaxy. For the major merger, we initialize two such disks on parabolic orbits with initial separation $R_{\text {init }}=5 R_{\text {vir }} / 8$ and pericentric distance twice the disk scale length (Robertson et al. $2006 \mathrm{~b})$. The orbit we focus on is the 'e' orbit of Cox et al. (2006a). We have checked that the differences between quiescent star formation and starbursts are insensitive to orbit as long as a strong starburst is induced (some orbits do not induce strong starbursts, but those are irrelevant for the purpose of studying the differences between starbursts and quiescent star formation), and the larger suite of simulations used to derive the fitting functions includes a variety of orbits.

\subsection{Radiative transfer}

Every 10 Myr we save snapshots of the GADGET2 simulations and use the 3-D Monte Carlo dust RT code SunRIsE 10 (Jonsson 2006; Jonsson et al. 2010) in post-processing to calculate the SEDs of the simulated galaxies. While we will summarize the key features of SunRISE here, we encourage the reader to see Jonsson (2006) and Jonsson et al. (2010) for full details. Except where noted, we use the fiducial parameters given in Jonsson et al. (2010). SunRISE calculates the emission from the stars and AGN in the GADGET-2 simulations and the attenuation and re-emission from dust. STARBURST99 (Leitherer et al. 1999) SEDs are assigned to all star particles according to their ages and metallicities. Star particles present at the start of the GADGET-2 simulation are assigned ages assuming that their stellar mass was formed at a constant rate equal to the star formation rate of the initial snapshot and gas and stellar metallicities via a closed-box model, $Z=-y \ln f_{g}$, where $f_{g}$ is the initial gas fraction and $y=0.02$. Black hole particles are assigned the luminosity-dependent templates of Hopkins et al. (2007) by assuming that the bolometric luminosity of a black hole particle is $L_{\mathrm{bol}}=0.1 \dot{M} c^{2}$, where $\dot{M}$ is the black hole accretion rate from the GADGET-2 simulations.

To calculate the dust density, and thus optical depth along a given line-of-sight, SUNRISE projects the GADGET-2 gas-phase metal density onto a 3-D adaptive mesh refinement grid using the SPH smoothing kernel. We have assumed $40 \%$ of the metals are in dust (Dwek 1998; James et al. 2002). We use a maximum refinement level of 10 , resulting in a minimum cell size of $55 h^{-1}$ pc. By performing runs with higher levels of refinement we have checked that the observed-frame submm flux density is converged to within $10 \%$. We use the Milky Way $\mathrm{R}=3.1$ dust model of Weingartner \& Draine (2001) as updated by Draine \& Li (2007). Dust models with different FIR opacity will lead to different relationships between submm flux density and dust mass, but we show how to rescale for different values of the opacity in Equation A9.

Once the dust density grid is constructed and the input sources are assigned SEDs, SUNRISE performs Monte Carlo RT by randomly emitting photon packets from the source particles and randomly drawing interaction optical depths from the appropriate probability distribution. We use $10^{7}$ photon packets total for each stage of the RT, having confirmed that this results in Monte Carlo noise of less than a few percent. The photon packets are scattered and absorbed by dust as they traverse the ISM. For each grid cell, the temperature of each dust species is calculated assuming the dust is in thermal equilibrium, and the dust re-emits the absorbed energy as a modified blackbody.

10 SUNRISE is publicly available 
A SUNRISE feature key to this work is its treatment of dust self-absorption. In high-density regions, the dust can be opaque to its own emission, so the contribution of the dust emission to dust heating must be computed in addition to the contribution from stars and AGN. SUNRISE computes the equilibrium dust temperatures self-consistently by iteratively performing the transfer of the dust emission and the temperature calculation using a reference field technique similar to that of Juvela (2005). (The details of the SUNRISE implementation are in Jonsson et al. 2010 and Jonsson \& Primack 2010.) This algorithm ensures accurate dust temperatures, and thus submm flux densities, even for the highly optically thick central starbursts.

The results of the SUNRISE calculation are spatially resolved, multi-wavelength (we use only 120 wavelengths here because of memory constraints) SEDs observed from 7 cameras distributed isotropically in solid angle, though in this paper we only utilize the integrated flux densities in the SCUBA (Holland et al. 1999) and AzTEC (Wilson et al. 2008) bands. For the purpose of calculating observed flux densities we assume the simulated galaxies are at redshift $z=2$.

\subsubsection{Differences from Narayanan et al.}

The primary difference between our simulations and those of N10 and Narayanan et al. (2010b) is the treatment of the ISM on sub-resolution scales. In order to model the effects of HII and photodissociation regions (PDRs), SUNRISE assigns star particles with ages less than 10 Myr SEDs from the HII region template library of Groves et al. (2008). The time-averaged fraction of solid angle obscured by the PDR, $f_{\mathrm{PDR}}$, strongly affects the resulting attenuation and IR emission (for a detailed discussion see Groves et al. 2008). Narayanan et al. assumed $f_{\mathrm{PDR}}=1$ (so that the young stars are completely obscured by PDRs for $10 \mathrm{Myr}$ ) in order to match the observed range of $850 \mu \mathrm{m}$ flux densities. Furthermore, they neglected the dust associated with the cold phase of the Springel \& Hernquist (2003) ISM model, typically $\gtrsim 30 \%$ of the total gas mass and $\gtrsim 90 \%$ of the gas mass in the central regions for snapshots classified as SMGs.

Motivated by concerns over applicability of the Groves et al. (2008) models to the extreme ISM densities and pressures encountered in our simulations, we set $f_{\mathrm{PDR}}=0$, eliminating all significant dust obscuration from the sub-resolution PDR model. Instead, we use the total gas density in the SPH simulations (i.e., both the diffuse and cold phases) to calculate the dust density. Since the dust mass implicit in the Groves et al. (2008) PDRs is not tied to the total dust mass of the simulated galaxy, it is possible that one can have more dust mass in the sub-resolution PDRs than is available in the galaxy. It is also possible that the sum of the dust mass in the PDRs is less than the total available in the cold phase of the sub-resolution ISM. Our treatment ensures that neither scenario can occur.

Our assumed ISM structure (cold phase volume filling factor of unity) is similar to what is observed for the dense cores of local ULIRGs (Scoville et al. 1991; Downes \& Solomon 1998; Sakamoto et al. 1999, 2008; Papadopoulos et al. 2010). Furthermore, it leads to effective far-IR optical depths (inferred from modified blackbody fitting using $L_{\nu} \propto\left(1-e^{-\tau_{\nu}}\right) B_{\nu}\left(T_{d}\right)$; see below for details) consistent with what is observed for local ULIRGs and SMGs, $\tau=1$ at rest-frame $\lambda \sim 200 \mu \mathrm{m}$ for the simulations versus at rest-frame $\lambda \sim 200-270 \mu \mathrm{m}$ for local ULIRGs (Lisenfeld et al. 2000; Papadopoulos et al. 2010; Rangwala et al. 2011) and SMGs (Lupu et al. 2010; Conley et al. 2011) (but cf. Kovács et al. 2010). However, it is still important to note that the sub-resolution ISM structure is the key uncertainty in these calculations (Younger et al. 2009a). While unresolved clumpy dust can significantly affect the resulting SED (Witt \& Gordon 1996; Városi \& Dwek 1999), a more detailed treatment is beyond the scope of this work. The trends presented in this work should be qualitatively insensitive to the sub-resolution ISM assumption because, as explained below, the dominant drivers of the differences between the quiescent star formation and starburst cases are the contribution from stars formed pre-burst to the luminosity at the time of the burst and the rapid gas consumption during the burst, both of which do not depend on the treatment of sub-resolution clumpy dust.

\section{RESULTS}

Figure 1 shows the time evolution of the observed SCUBA $850 \mu \mathrm{m}$ flux density $(\mathrm{mJy}), \operatorname{SFR}\left(M_{\odot} \mathrm{yr}^{-1}\right.$; calculated by dividing the mass of stars formed in the last 10 Myr of the simulation by $10 \mathrm{Myr}$ ), bolometric luminosity $L_{\mathrm{bol}}\left(L_{\odot}\right)$, initial fraction of baryonic mass that is gas $f_{g}$, dust mass $M_{d}\left(M_{\odot}\right)$, and dust temperature 11$] T_{d}(\mathrm{~K})$ for the isolated disk galaxy (left) and merger (right), where all quantities except $f_{g}$ have been normalized by dividing by their maximum values, given in the legend. When calculating the observed flux density we have assumed the simulated galaxy is at $z=2$. The disk is somewhat unstable initially; after the disk settles, $L_{\text {bol }}$, SFR, and $S_{850}$ decrease steadily with time. Over the 2 Gyr of the simulation the gas fraction decreases from $60 \%$ to $20 \%$. As the gas is consumed, the SFR, and thus $L_{\mathrm{bol}}$, both decrease, by factors of $\sim 10$ and $\sim 5$, respectively. $S_{850}$ decreases by $\sim 2.5 \mathrm{x} . M_{d}$ also decreases as a result of the decrease in gas mass, but only by $\sim 40 \%$ because the decrease in gas mass is partially mitigated by metal enrichment of the gas from star formation, as the metallicity doubles over the course of the simulation. While it may seem counter-intuitive that $M_{d}$ decreases with time, for a simple closed-box model assuming dust traces metals and constant yield it can be shown (Edmunds \& Eales 1998) that for $f_{g} \lesssim 0.6$ the dust mass increases at most by $\sim 0.1 \mathrm{dex}$, and it decreases monotonically with $f_{g}$ for $f_{g} \lesssim 0.4$. Furthermore, the preferential consumption

11 We have calculated $T_{d}$ by fitting the modified blackbody $L_{\nu} \propto$ $\left(1-\exp \left[-\left(\nu / \nu_{0}\right)^{\beta}\right]\right) B_{\nu}\left(T_{d}\right)$ to the rest-frame $20-1000 \mu \mathrm{m}$ SED, allowing all parameters to vary. Here $\nu_{0}$ is the frequency at which the effective optical depth $\tau_{\nu}=1$. We have assumed the opacity has a power-law dependence on $\nu$ in the IR, $\kappa_{\nu} \propto \nu^{\beta}$. Note we have not used the optically thin form $L_{\nu} \propto \nu^{\beta} B_{\nu}\left(T_{d}\right)$, as is almost always done, because we find that the first form, which does not assume optical thinness, provides a significantly better fit to our SEDs. This is because our simulated SMGs can be optically thick out to rest-frame $\gtrsim 100 \mu \mathrm{m}$, which is supported by recent Herschel observations from Lupu et al. (2010) and Conley et al. (2011), who found $\tau \sim 1$ at rest-frame $\lambda \sim 200 \mu \mathrm{m}$. Our fitting procedure gives systematically higher $T_{d}$ (by as much as $20 \mathrm{~K}$ ) than when the optically thin form is used, so comparisons of our dust temperatures to other results should take this into account. 

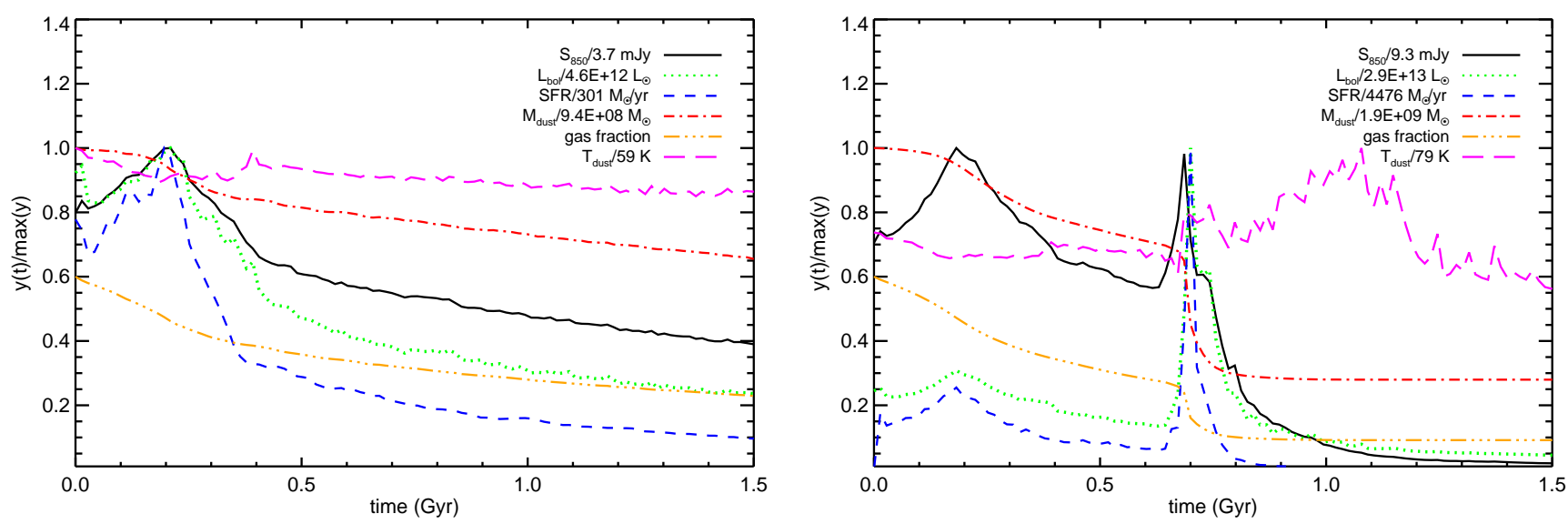

Figure 1. Left: The isolated disk simulation's observed-frame integrated SCUBA $850 \mu \mathrm{m}$ flux density (mJy, assuming $z=2$; solid black) measured from one of the seven viewing angles, bolometric luminosity $\left(L_{\odot} ;\right.$ dotted green), SFR $\left(M_{\odot} \mathrm{yr}^{-1} ;\right.$ dashed blue), dust mass $M_{d}$ $\left(M_{\odot}\right.$; dash dot red), gas fraction $f_{g}$ (dash dot dot dot orange), and dust temperature (derived from SED fitting) $T_{d}$ (K; long dashed magenta) versus time (Gyr). Except for $f_{g}$, the quantities have been normalized by dividing by their maximum values, given in the legend. Once the disk reaches equilibrium, $S_{850}, L_{\mathrm{bol}}$, SFR, and $f_{g}$ concomitantly decrease exponentially with time as the gas is converted into stars. $M_{d}$ also decreases, but by less than a factor of 2 , because the decreasing $f_{g}$ is offset by the increasing metallicity of the gas. $T_{d}$ decreases from $\sim 60 \mathrm{~K}$ to $\sim 50 \mathrm{~K}$. Right: Same, but for the major merger simulation. All quantities are totals for the two-disk system. Compared to the isolated disk the time evolution is more complex: Initially $S_{850}, \mathrm{SFR}, L_{\mathrm{bol}}$, and $M_{d}$ are roughly just the sums of the values for the isolated progenitor disks. At first passage $(t \sim 0.1 \mathrm{Gyr})$, the SFR is not elevated much beyond the baseline rate because the disks are very gas-rich and thus lack the massive stellar bar needed to efficiently remove angular momentum from the gas (Hopkins et al. 2009b). As the two disks coalesce $(t \sim 0.7 \mathrm{Gyr})$, tidal torques cause a burst of star formation, resulting in the sharp increase in the SFR $(\gtrsim 16 \mathrm{x}), L_{\mathrm{bol}}(\sim 7 \mathrm{x})$, and $S_{850}(\lesssim 2 \mathrm{x})$ at $\sim 0.7 \mathrm{Gyr}$. The increase in $L_{\mathrm{bol}}$ is much less than that boost in SFR because the luminosity of the stars formed during the burst is only $\sim 6 \mathrm{x}$ the luminosity from stars already formed pre-burst. $T_{d}$ increases sharply from $\sim 50 \mathrm{~K}$ to $\sim 65 \mathrm{~K}$ because of the strong increase in $L_{\mathrm{bol}}$, concurrent decrease in $M_{d}$, and more compact geometry. This mitigates the increase in $S_{850}$ that occurs from increased $L_{\mathrm{bol}}$. The second, minor peak in $L_{\mathrm{bol}}$, which occurs $\sim 40 \mathrm{Myr}$ after the peak SFR, corresponds to the peak AGN luminosity. Pre-coalescence, $f_{g}$ decreases at a rate similar to the isolated disk case. At coalescence the gas is rapidly consumed in the central starburst. $M_{d}$ decreases by a factor of 4.5 , with the bulk of the decrease occurring at coalescence.

of metal-enriched gas that occurs in our models should result in a lower dust mass than the simple closed-box case of Edmunds \& Eales (1998), which assumes perfect mixing.

The behavior of the merger (Figure 1, right) is qualitatively different from that of the isolated disk. Initially, SFR, $L_{\mathrm{bol}}$, and $S_{850}$ are roughly equal to the sum of the isolated values for the two progenitor disks, because the disks are too gas-rich at first passage $(t \sim 0.1 \mathrm{Gyr})$ for tidal torques to cause a strong starburst, as a significant stellar bar is required for the gas to efficiently loose angular momentum (Hopkins et al. 2009b). However, at final coalescence of the two disks $(\sim 0.7 \mathrm{Gyr})$ tidal torques induce a strong starburst, causing the SFR to increase by a factor of $\gtrsim 16$. The peak of the burst is very narrow and significant luminosity from previously formed stars remains, so the bolometric luminosity increases by a much smaller amount $(\sim 7 \mathrm{x})$ than the SFR. Moreover, as the gas is rapidly consumed in the starburst, $M_{d}$ plummets by a factor of 3 . Along with the more compact geometry, the decreased dust mass causes the SED to become hotter, with $T_{d}$ increasing from $\sim 50 \mathrm{~K}$ to $\sim 65 \mathrm{~K}$. The increase in dust temperature during the starburst is qualitatively consistent with observations, as local ULIRGs (i.e., merger-induced starbursts) tend to have hotter dust temperatures $(\sim 42 \mathrm{~K})$ than less luminous (quiescent) galaxies $(\sim 35 \mathrm{~K})$ (Clements et al. 2010). The increased $T_{d}$ partially offsets the increase in $S_{850}$ caused by the increased luminosity. The combination of the significant pre-burst contribution to $L_{\mathrm{bol}}$, the small mass of stars formed in the burst, and the increased $T_{d}$ cause $S_{850}$ to increase by $\lesssim 2 \mathrm{x}$ even though the SFR increases by $\gtrsim 16 \mathrm{x}$ in the burst.

\subsection{The relationship between submm flux density and} SFR

Figure 2 shows the observed SCUBA $850 \mu \mathrm{m}$ flux density in mJy versus star formation rate in units of $M_{\odot} \mathrm{yr}^{-1}$ for the isolated disk (left) and major merger (right) viewed from all of the 7 cameras. The submm flux density of the isolated disk is tightly correlated with SFR, increasing monotonically as SFR ${ }^{0.4}$ (see best-fit curve). This correlation occurs because once the disk settles, $L_{\mathrm{bol}}$ and SFR both decrease exponentially with time. The dust mass also decreases, but by less than a factor of 2 over the 2 Gyr of the simulation (see Figure 1). Both the decreased luminosity and the decreased dust mass cause the submm flux density to decrease.

The case for the major merger is again qualitatively different. Pre-coalescence, the relationships are essentially the same as for the isolated disks. This is because $S_{850}$, SFR, $L_{\text {bol }}$, and $M_{d}$ at this stage are essentially just the sum of the two disks' isolated values, and multiplying all quantities by the same factor ( 2 for the major merger here) does not change the power-law index. The normalization of the relation is $\sim 1.5$ greater for the merging disks than for the isolated disk. The reason is as follows: An isolated disk of SFR $s$ has $S_{850}=A s^{0.4}$, where $A$ is the normalization of the SFR- $S_{850}$ relation for the isolated disk. For a non-interacting system of two identical disks to have total SFR equal to that of the single isolated disk, the two disks must each have $\mathrm{SFR}=0.5 \mathrm{~s}$. Thus the total submm flux density of the system is the submm flux density of a single disk of SFR $0.5 \mathrm{~s}$, which 

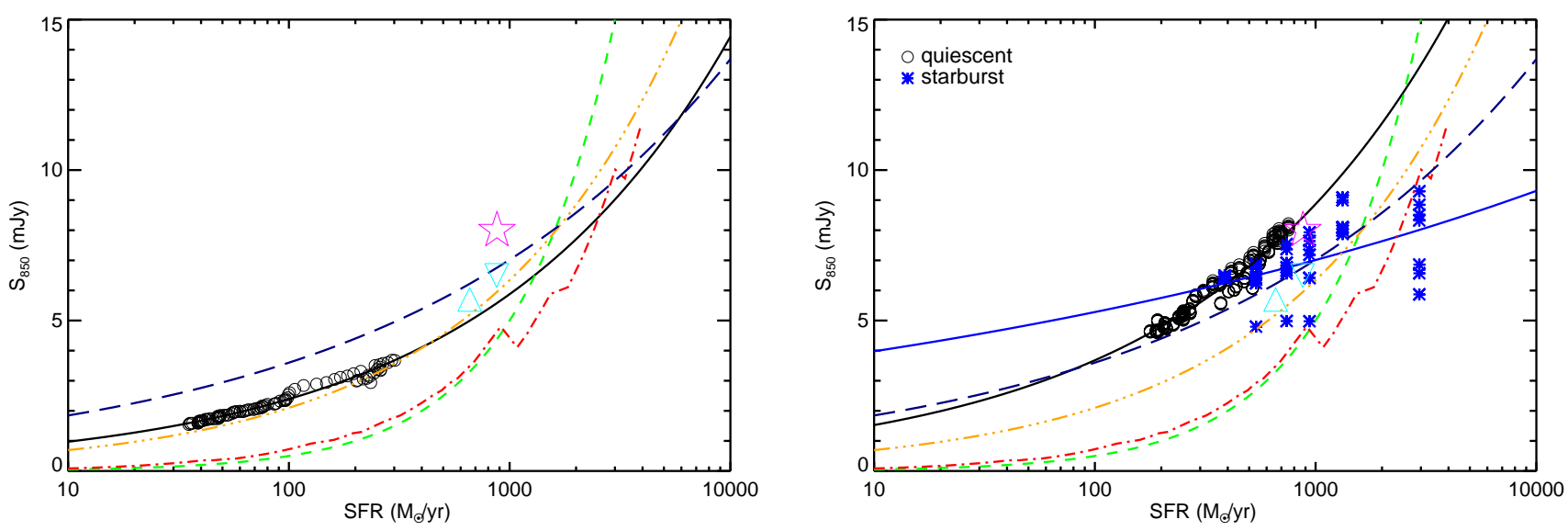

Figure 2. Integrated SCUBA $850 \mu \mathrm{m}$ flux density (mJy) versus SFR $\left(M_{\odot} \mathrm{yr}^{-1}\right)$ for the isolated disk $($ left $)$ and major merger $($ right $)$ viewed from all of the 7 different cameras. The best-fit power laws (solid lines), linear relation $S_{850}=0.5 \mathrm{mJy}\left(\mathrm{SFR} / 100 M_{\odot} \mathrm{yr}^{-1}\right.$ ) (green dashed lines; normalization from Neri et al. 2003 and Equation A11), the relation for the Charv \& Elbaz (2001) templates (red dash dot), the Magnelli et al. (2010) relations for their entire sample (orange dash dot dot dot) and excluding the lensed SMGs (navy long-dashed), the value for the Pope et al. (2008) composite SED (magenta star), and the median (cyan upward-pointing triangle) and mean (cyan downward-pointing triangle) from Michałowski et al. (2010a) are also shown. For the isolated disk, the submm flux density is tightly correlated with both SFR and $L_{\mathrm{bol}}$, increasing essentially monotonically as SFR ${ }^{0.4}$. For the major merger, pre-coalescence (black open circles) the power-law index is the same as for the isolated disks because the SFR, $L_{\mathrm{bol}}$, dust mass, and submm flux density are essentially two times the isolated disk values (see Figure 1), so only the normalization changes. During the coalescence-induced starburst (blue asterisks), the relationship is significantly shallower, with submm flux density scaling as $\mathrm{SFR}^{0.1}$. This is due to two effects: 1 . The stars formed before the peak of the starburst contribute significantly to $L_{\mathrm{bol}}$ at the starburst peak, so $L_{\mathrm{bol}} \not<$ SFR. 2. The rapid gas consumption during the burst causes $M_{d}$ to plummet, and the decrease in dust mass and more compact geometry cause $T_{d}$ to increase sharply, mitigating the increase of $S_{850}$ caused by the increased $L_{\mathrm{bol}}$.

we calculate using the isolated relation, multiplied by 2 . This is $S_{850}=2 A(0.5 s)^{0.4}=1.5 A s^{0.4}$. Therefore the normalization of the SFR- $S_{850}$ relation for the sum of two identical disks is 1.5 times that of the individual disk relation. This fact has important implications for the SMG population, which we discuss in 4.2 .

On the other hand, the merger-induced burst is significantly less effective at boosting the submm flux density. For a given SFR, the submm flux density is significantly less than for the isolated and pre-coalescence (quiescent star formation) cases. This is because of two reasons: 1. The sharp decrease in dust mass and more compact geometry cause an increase in dust temperature, mitigating the increase in $S_{850}$ caused by increased $L_{\mathrm{bol}} .2$. The significant luminosity contributed by stars formed before the starburst causes $L_{\text {bol }}$ to increase sub-linearly with SFR 12 During the burst, $L_{\text {bol }} \approx L_{\text {pre-peak }}+\alpha \mathrm{SFR}$, where $\alpha$ is the luminosity per unit star formation rate for an instantaneous burst. Thus $L_{\text {bol }}$ is not proportional to SFR when $L_{\text {pre-peak }}$ is non-negligible compared to the luminosity of newly-formed stars, which is the case here because a relatively small fraction of the stellar mass is formed in the sharp, short-duration burst. For the burst, the submm flux density scales as $\mathrm{SFR}^{0.1}$ (see best-fit curve), significantly more weakly than for quiescent star formation, and the ratio of submm flux density to SFR is significantly lower. Hence, bursts of star formation are significantly less effective at boosting submm flux density than one might naively expect.

It is interesting to note that, during the starburst, the observed submm flux density can vary significantly with

\footnotetext{
12 In principle the AGN can also cause such an effect, but for snapshots classified as SMGs the typical AGN contribution to the IR luminosity is $\lesssim 10 \%$, so the AGN is sub-dominant.
}

viewing angle (e.g., for the snapshot with peak SFR, $S_{850}$ varies in the range $\sim 6-9 \mathrm{mJy}$ depending on the camera). We have confirmed that this variation is due to dust self-absorption: the central regions of the starburst can be so obscured that even the IR emission is significantly anisotropic. As a result, the dust temperature, and thus submm flux density, depends on the line-ofsight. Though we will not explore this possibility further in this work, we note that differences in viewing angle may be enough to account for the spread of dust temperatures observed for high-z ULIRGs. In other words, from one viewing angle a simulated galaxy may be identified as an SMG whereas from another viewing angle the same galaxy could be identified as a hot-dust-dominated ULIRG undetected in the submm.

Figure 2 also shows the linear relation $S_{850}=$ $0.5 \mathrm{mJy}\left(\mathrm{SFR} / 100 M_{\odot} \mathrm{yr}^{-1}\right)$ (green dashed lines; obtained using the $S_{850}-L_{\mathrm{IR}}$ relation from Neri et al.|2003 and Equation A11), the relation for the Chary \& Elbaz (2001) templates (red dash dot), the Magnelli et al. (2010) relations for their entire sample (orange dash dot dot dot) and excluding the lensed SMGs (navy long-dashed), the value for the Pope et al. (2008) composite SED (magenta star), and the median (cyan upward-pointing triangle) and mean (cyan downwardpointing triangle) from Michałowski et al. (2010a). The Chary \& Elbaz (2001) and Pope et al. (2008) values were obtained by redshifting the templates to $z=2$ and converting $L_{\mathrm{IR}}$ of each template to SFR using Equation A11. We used Equation A11 to convert the Magnelli et al. (2010) relations from $L_{\mathrm{IR}}$ to SFR.

The typical values from Pope et al. (2008) and Michałowski et al. (2010a) are consistent with the data from our major merger simulation. As explained above, the relations we find are much shallower than linear, 
so the Neri et al. (2003) relation differs significantly from our relations for both the quiescent and starburst modes. The Chary \& Elbaz (2001) templates are also very discrepant because high-redshift ULIRG SEDs are often better fit by local templates appropriate for less luminous, colder galaxies (e.g., Pope et al. 2006; Dannerbauer et al. 2010; Rex et al. 2010). The Magnelli et al. (2010) relations agree better with our simulations: for the full sample, $S_{850} \propto \mathrm{SFR}^{0.48}$. This is a slightly steeper relation than what we find for quiescent disks and significantly steeper than that for starbursts. When the six lensed SMGs are removed from their sample, Magnelli et al. find $S_{850} \propto \mathrm{SFR}^{0.29}$. The powerlaw index of this relation is less than that for our quiescent disks but greater than that for our starbursts. The lensed SMGs tend to be intrinsically fainter and thus less likely to be strong starbursts than the non-lensed population, so it is reasonable that inclusion of the lensed SMGs leads to a steeper relation. While it is interesting that the Magnelli et al. relation for the unlensed SMGS is crudely consistent with what we expect for a mixed population, one should not over-interpret this comparison. As we will discuss below, $S_{850}$ cannot be determined solely from SFR because the dust mass plays a significant role also. However, a robust conclusion that should be drawn from Figure 2 is that, in the simulations, the starburst mode is less efficient at boosting submm flux than the quiescent mode.

\subsection{Dependence of submm flux density on $S F R, L_{\mathrm{bol}}$, and $M_{d}$}

For a given SFR, galaxies of different masses tend to also have different dust masses; thus the normalization of the $S_{850}$-SFR relation varies for different mass simulations though the scalings are similar. As a result, one cannot calculate the submm flux density given only the SFR, but it is possible to parameterize the submm flux density as a function of SFR and dust mass. Since much of the discrepancy in the $S_{850}$-SFR relations for quiescent star formation and starbursts is caused by the rapid decrease in dust mass during the starburst, we expect that including dust mass in our parameterization will eliminate much of the difference between quiescent and starburst star formation modes. We have analyzed the full set of simulations from our SMG number counts work (Hayward et al. 2011, C. Hayward et al. 2011, in preparation), which includes a range of progenitor disk baryonic masses $\left(\sim 3.5 \times 10^{10}-4 \times 10^{11} M_{\odot}\right)$, mass ratios $(\sim 0.1-1)$, and initial gas fractions $(0.6-0.8)$, fitting the submm flux density as a power law in both SFR and dust mass. Both the quiescent and starburst phases are included. Perhaps surprisingly, the following relations (for simulated galaxies placed at redshift $z=2$ ) hold to within $\sim 0.1$ dex for all but a few outliers over the range $0.5 \mathrm{mJy} \lesssim S_{850} \lesssim 15 \mathrm{mJy}:$

$$
\begin{aligned}
S_{850} & =0.65 \mathrm{mJy}\left(\frac{\mathrm{SFR}}{100 M_{\odot} \mathrm{yr}^{-1}}\right)^{0.42}\left(\frac{M_{d}}{10^{8} M_{\odot}}\right)^{0.58}(1) \\
S_{1.1} & =0.30 \mathrm{mJy}\left(\frac{\mathrm{SFR}}{100 M_{\odot} \mathrm{yr}^{-1}}\right)^{0.36}\left(\frac{M_{d}}{10^{8} M_{\odot}}\right)^{0.61},
\end{aligned}
$$

where $S_{850}$ and $S_{1.1}$ are the fluxes in the SCUBA $850 \mu \mathrm{m}$ and AzTEC $1.1 \mathrm{~mm}$ bands, respectively.
Note that the SFR exponent in these relations is similar to that for the $S_{850}$-SFR relation for quiescent $\mathrm{SF}$ $(\sim 0.4)$. As explained in \$3.1, the sharp decrease in dust mass during the starburst is one of the main reasons the $S_{850-\mathrm{SFR}}$ relation is much shallow for starbursts than for quiescent SF. Adding $M_{d}$ as a parameter effectively decouples this effect; in other words, for fixed dust mass the $S_{850-\mathrm{SFR}}$ relations for the two SF modes are much more similar than when the evolution of the dust mass is taken into account. Remaining differences caused by the contribution from stars formed pre-burst to the burst luminosity, AGN contribution, geometry, and other factors prevent Eq. (1) from recovering $S_{850}$ exactly, but the small scatter suggests that these factors are subdominant.

We can also fit the submm flux density as a function of $L_{\mathrm{bol}}$ and $M_{d}$ :

$$
\begin{aligned}
S_{850} & =0.40 \mathrm{mJy}\left(\frac{L_{\mathrm{bol}}}{10^{12} L_{\odot}}\right)^{0.52}\left(\frac{M_{d}}{10^{8} M_{\odot}}\right)^{0.60} \\
S_{1.1} & =0.20 \mathrm{mJy}\left(\frac{L_{\mathrm{bol}}}{10^{12} L_{\odot}}\right)^{0.46}\left(\frac{M_{d}}{10^{8} M_{\odot}}\right)^{0.63} .
\end{aligned}
$$

These fitting functions are accurate to within $\sim 0.15$ dex. Replacing $L_{\text {bol }}$ with the bolometric IR luminosity $L_{\mathrm{IR}}$ yields a similar result. Figure 3 shows how well these fitting functions reproduce the submm flux density of our simulated galaxies. It is discussed in more detail in \$3.2.2.

\subsubsection{Relations for an optically thin modified blackbody}

It is instructive to compare the above relations to those for a single-temperature mass of dust transparent to its own emission, the model which is implicit in the standard method of fitting a modified blackbody to the IR SED. For a mass of dust in thermal equilibrium with temperature $T_{d}$ we can express the submm flux density as a function of dust bolometric luminosity $L_{d}$ and dust mass $M_{d}$ or SFR and $M_{d}$. Assuming $z=2$ and far-IR spectral index $\beta=2$, the relations are (see the Appendix for a derivation)

$$
S_{850}=1.4 \mathrm{mJy}\left(\frac{\mathrm{SFR}}{100 M_{\odot} \mathrm{yr}^{-1}}\right)^{1 / 6}\left(\frac{M_{d}}{10^{8} M_{\odot}}\right)^{5 / 6}
$$

and

$$
S_{850}=1.4 \mathrm{mJy}\left(\frac{L_{d}}{10^{12} L_{\odot}}\right)^{1 / 6}\left(\frac{M_{d}}{10^{8} M_{\odot}}\right)^{5 / 6} .
$$

These should be compared to Equations (11) and (2), respectively, by assuming $L_{\text {bol }} \approx L_{d}$ (i.e., the luminosity emitted by stars and AGN is completely absorbed by dust), which is a reasonable approximation for snapshots classified as SMGs $\left(S_{850}>3 \mathrm{mJy}\right)$. The submm flux depends only weakly on redshift for the redshift range of interest $(1 \lesssim z \lesssim 5)$, scaling as $(1+z)^{\beta-1} D_{A}^{-2}$, where $D_{A}$ is the angular diameter distance at redshift $z$ (see Equation A9.

\subsubsection{Comparison of the relations to the full radiative transfer}



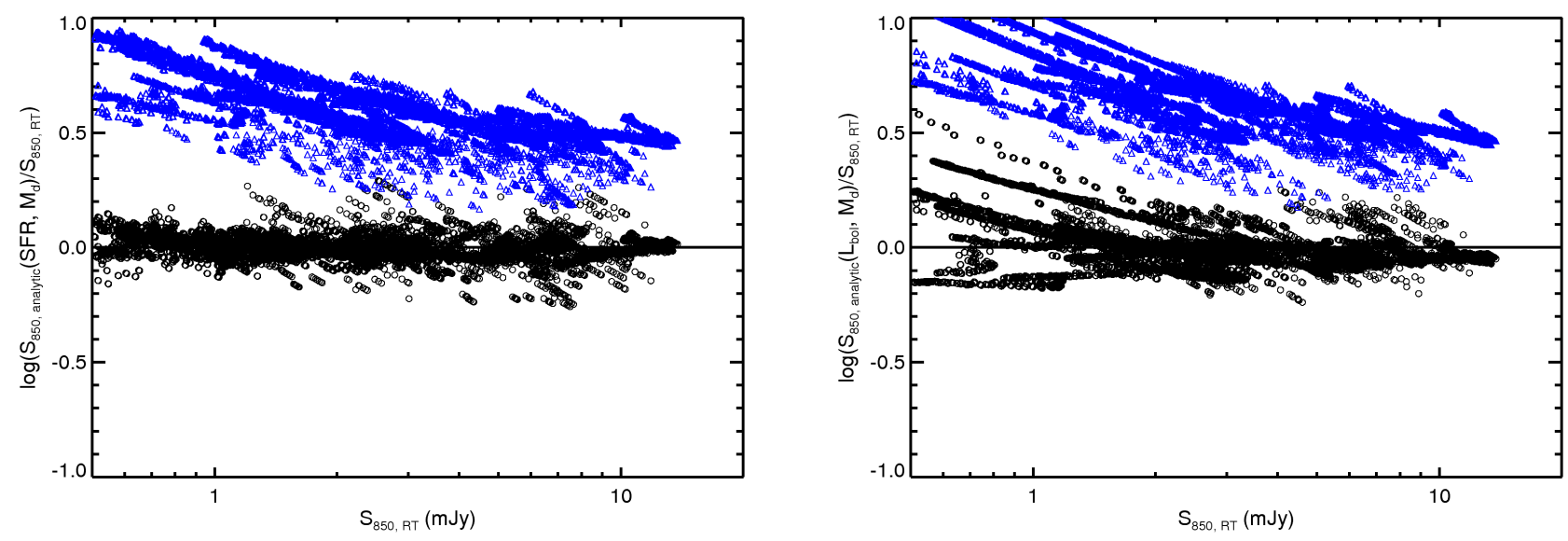

Figure 3. Left: Logarithm of the ratio of the submm flux density calculated using one of the analytic forms $\left(S_{850, \text { analytic }}\right)$ to the submm flux density calculated through the full RT $\left(S_{850, \mathrm{RT}}\right)$ vs. $S_{850, \mathrm{RT}}$ for all time snapshots of our simulated galaxies. Black circles show the

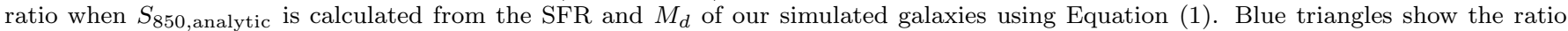
when the optically thin modified blackbody model (Equation 3) is used. The line $S_{850 \text {, analytic }}=S_{850 \text {, RT }}$ is shown to guide the eye. Right: Same, except now the black circles show the values when Equation (2) is used to calculate the submm flux density from $L_{\mathrm{bol}}$ and $M_{d}$ of the simulation snapshots, and the blue triangles show the values when Equation (4) is used, assuming $L_{\mathrm{bol}} \approx L_{d}$. In both cases the simple optically thin modified blackbody model overpredicts the submm flux density by $\gtrsim 0.3$ dex for the simulated SMGs, and the overprediction is worse for lower $S_{850}$.

Figure 3 shows the logarithm of the ratio of the submm flux density calculated using the above equations $\left(S_{850, \text { analytic }}\right)$ to the submm flux density calculated through the RT ( $\left.S_{850, \mathrm{RT}}\right)$ versus $S_{850, R T}$. The left panel shows the results when Equations (1) black circles) and (3. blue triangles) are used to calculate $S_{850}$ from SFR and $M_{d}$. The right panel shows the results when $L_{\mathrm{bol}}$ and $M_{d}$ are used instead, with the black circles corresponding to Equation (2) and the blue triangles Equation (4). The fitting functions derived from the simulations are able to reproduce $S_{850}$ from $\operatorname{SFR}\left(L_{\mathrm{bol}}\right)$ and $M_{d}$ to within $\sim 0.1$ (0.15) dex. The simple modified blackbody model tends to overpredict the submm flux density by $\gtrsim 0.3$ dex; the typical over-prediction is $\sim 0.5$ dex (a factor of 3). (Note that the corresponding uncertainties in SFR, $L_{\text {bol }}$, and $M_{d}$ for fixed observed submm flux density would be less because the submm flux density scales with these quantities sublinearly.) Furthermore, the error in the prediction correlates with $\operatorname{SFR}\left(L_{\mathrm{bol}}\right)$ and dust mass because of the differences in the power-law indices for the fitting functions and the modified blackbody relations.

The optically thin modified blackbody model fails for multiple reasons. From SED fitting we find that the simulated galaxies can have effective optical depth $\tau>1$ out to rest-frame $\sim 200 \mu \mathrm{m}$. For fixed dust temperature, the optically thin assumption will result in an overestimate of the luminosity density at wavelengths for which $\tau \gtrsim 1$ because $\left(1-\exp \left[-\left(\nu / \nu_{0}\right)^{\beta}\right]\right)<\left(\nu / \nu_{0}\right)^{\beta}$ for all $\nu>0$. Thus Equation (A3) will overestimate $L_{d}$ for fixed $T_{d}$ and $M_{d}$. If $L_{d}$ and $M_{d}$ are fixed, the dust temperature will be underestimated when optical thinness is assumed, and, therefore, the submm flux density will be overestimated. Also, $L_{d} \approx L_{\mathrm{bol}}$ is less accurate an approximation for the faintest sources than for the brightest because $L_{d} / L_{\mathrm{bol}}$ increases with $L_{\text {bol }}$ (Jonsson et al. 2006). If $L_{d}<L_{\text {bol }}$, the assumption that $L_{d} \approx L_{\text {bol }}$ will overestimate $L_{d}$ and thus overestimate the submm flux density; this may explain why the overprediction is worse for lower $S_{850}$. Fi- nally, we have seen above that the assumption $L_{\text {bol }} \propto$ SFR is invalid during the burst.

\section{DISCUSSION}

We have demonstrated that the submm flux density of a galaxy scales differently with SFR for quiescent star formation and starbursts. The ratio of sub-mm flux density to SFR is significantly less for merger-induced bursts than for quiescent star formation. This is because of the rapid decrease in dust mass and more compact geometry during the starburst, which causes the SED to become hotter, and the significant contribution from stars formed pre-burst to the luminosity during the burst, which makes the luminosity increase by a much smaller factor than the SFR. As a result, merger-induced starbursts are less efficient at boosting submm flux density than one might naively expect.

Our results have a number of important implications; we discuss these now.

\subsection{Predicting submm flux densities from models}

One implication of this study is that, at a fixed redshift, the galaxies with highest submm flux density are not necessarily those with the highest bolometric luminosities or SFRs. Thus theoretical models, be they simulations or semi-analytic, must explicitly calculate the submm flux density of their simulated galaxies in order to select which are SMGs as opposed to simply selecting the most rapidly star-forming or most luminous objects. However, the computational expense required to self-consistently calculate the submm flux density limits this approach to idealized, non-cosmological simulations (as done here), individual galaxies excised from cosmological zoom-in simulations, or semi-analytic models in which various simplifying assumptions must be made. As an alternative to performing RT, cosmological simulations and semi-analytic models can use the relations among submm flux density, SFR or bolometric luminosity, and dust mass presented herein (Equations 1 and 2) 
to assign submm flux density to simulated galaxies. Additionally, observers can use the relations to estimate the instantaneous SFR given submm flux density and dust mass (obtained from fitting the IR SED using the full modified blackbody as we have done or by measuring the gas mass and assuming a dust-to-gas ratio; ignoring uncertainties on submm flux density, a dust mass accurate to a factor of 2 gives SFR accurate to a factor of $3)$.

\subsection{Heterogeneity of the $S M G$ population}

These results also imply that the SMG population is heterogeneous. We have seen that it is possible for a quiescently star-forming disk to have submm flux density equal to that of a merger with much higher SFR (Figure 2). Furthermore, since the scaling of submm flux density with SFR is sublinear, adding two equal disks (and thus doubling the dust mass and SFR of the system) increases the submm flux density more than simply boosting the SFR by a factor of 2; Equation (1) shows that SFR would have to be boosted by $5 \mathrm{x}$ to achieve a $2 \mathrm{x}$ boost in submm flux density if dust mass is kept constant. When the sharp increase in dust temperature during the starburst and the narrowness of the burst are accounted for the effect becomes even stronger: in Figure 1] we see that a $\gtrsim 16 \mathrm{x}$ increase in SFR gives $\mathrm{a} \lesssim 2 \mathrm{x}$ increase in submm flux density.

The single-dish submm telescopes used for wide-field surveys of SMGs have beam sizes $\gtrsim 15^{\prime \prime}(\gtrsim 130 \mathrm{kpc}$ at $z=2$ ). Thus, during a merger the two progenitor galaxies will spend a considerable amount of time within the area of the beam. From the above arguments, we see that this is a very efficient way to create an SMG, but this contribution has been relatively unappreciated. We argue that the SMG population attributable to mergers is bimodal: some are merger-induced starbursts and some are two (or more) infalling disks (normal galaxies that are not yet interacting strongly) blended into one submm source ("galaxy-pair SMGs") 13 Note also that not only major mergers but also favorably oriented minor mergers (see, e.g., Cox et al. 2008; Hopkins et al. 2009b) can contribute to the SMG population.

Furthermore, the most massive, rarest 'isolated disks', and even small groups, may also contribute; we expect this contribution to be subdominant because SMGs are on the exponential tail of the mass function, but we defer a precise determination of their contribution to future work. Though both the merger-induced starburst and galaxy-pair populations are mergers, only in the former is the star formation merger-driven (and only partially, as the baseline star formation that would occur in the disks even if they were not interacting is significant).

\footnotetext{
13 Wang et al. (2011) recently presented high-resolution submm continuum images of two SMGs which were previously identified as single sources but are resolved as 2 or 3 distinct submm sources in their images. The sources are at significantly different redshifts and thus physically unrelated. Our galaxy-pair SMGs are also two sources blended into one, but they are distinct from the type of SMGs Wang et al. observed because they are merging and thus physically connected. Both types of blended sources are potentially important SMG subpopulations that complicate our understanding of SMGs, and it is crucial to understand the relative contributions of merger-induced starbursts, galaxy pairs/infall-stage mergers, quiescently star-forming disks, and physically unrelated, blended sources.
}

Given that the physically meaningful property of local ULIRGs is not that their IR luminosities are $\geq 10^{12} L_{\odot}$ but that they are powered by merger-driven star formation and AGN, only the merger-driven starburst category of SMGs should be considered physically analogous to local ULIRGs.

The observational signatures and physical implications of this bimodality will be discussed in future work. Here we simply note that the galaxy-pair contribution is supported observationally by the frequency of multiple radio (Ivison et al. 2002, 2007; Chapman et al. 2005; Younger et al. 2009c), $24 \mu \mathrm{m}$ (Pope et al. 2006), and $350 \mu \mathrm{m}$ (Kovács et al. 2010) counterparts to SMGs; by CO interferometry showing that a large fraction of SMGs are resolved binaries (Tacconi et al. 2006, 2008; Bothwell et al. 2010; Engel et al. 2010); and by the SMGs that have morphologies that do not resemble merger remnants (e.g., Bothwell et al. 2010; Carilli et al. 2010; Ricciardelli et al. 2010; Targett et al. 2011) 14

\section{3. $S M G$ masses}

The masses of SMGs are hotly debated, with different authors finding masses discrepant by $\sim 6 \mathrm{x}$ for the same SMGs (Michałowski et al. 2010a, b; Hainline et al. 2010). Accurate masses are important in order to test potential evolutionary relationships among SMGs and other galaxy classes (e.g., Brodwin et al. 2008; Bussmann et al. 2009a, b; Narayanan et al. 2010b; Rothberg \& Fischer 2010) and to check that number densities of SMGs are consistent with observed stellar mass functions. Stellar mass determinations from SED fitting are limited by uncertainties in stellar evolution tracks, the initial mass function, star formation histories, dust attenuation, and AGN contamination. Since our models use star formation histories, attenuation (from the geometry of stars, AGN, and dust), and AGN components that originate directly from the hydrodynamic simulations instead of the standard assumptions - e.g., instantaneous burst or exponential star formation histories, the Calzetti attenuation law (Calzetti et al. 1994, 2000; Calzetti 1997) — we can provide constraints on SMG stellar masses that are complementary to those derived from SED fitting.

Given the inefficiency of bursts at boosting submm flux density that we have demonstrated above, SMGs must be very massive, because smaller galaxies undergoing even very strong bursts cannot make SMGs. Our models require $M_{\star} \gtrsim 6 \times 10^{10} M_{\odot}$ to reach $S_{850} \gtrsim 3 \mathrm{mJy}$, and typical masses are higher. The area of the $S_{850}-M_{\star}$ plot spanned by our models agrees well with the observationally derived values of Michałowski et al. (2010a). About half of the Hainline et al. (2010) values lie in the area spanned by our models, whereas the other half have lower masses. However, the single-component star formation histories assumed by Hainline et al. may cause the stellar masses to be underestimated by $\sim 2 \mathrm{x}$, which would resolve much of the discrepancy. A detailed comparison of the mass estimates will be presented in $\mathrm{M}$. Michałowski et al. (2011, submitted).

14 Note, however, that the Bothwell et al. (2010) and Carilli et al. (2010) objects that resemble disk galaxies may in fact be the molecular disks that re-form rapidly after a gas-rich merger (Narayanan et al. 2008b; Robertson \& Bullock 2008). 


\subsection{SMG duty cycles}

Understanding the duty cycle of SMGs is important for predicting submm counts from models, quantifying the contribution of SMGs to stellar mass buildup, and interpreting star formation efficiencies of SMGs. Since the submm flux density depends on luminosity heating the dust, dust mass, and geometry, the submm duty cycle depends on the same factors. As we have seen, the starburst induced at merger coalescence causes a sharp peak in SFR, $L_{\mathrm{bol}}$, and submm flux density. However, the duty cycle is limited because of the sharp cutoff in SFR, and thus drop in $L_{\mathrm{bol}}$, after the burst and the significant drop in dust mass that occurs as highly enriched gas is consumed in the burst.

Since the submm flux density depends more strongly on dust mass than either SFR or $L_{\mathrm{bol}}$ (see Equations 1 and 2), it is more efficient if one can keep more dust around at the expense of lower star formation rate. The quiescent star formation mode does exactly this. As a result, the galaxy-pair phase (discussed in 44.2) adds significantly to the submm duty cycle. Figure 1 shows that the galaxy-pair phase has a longer submm duty cycle than the burst, though the lack of smooth accretion in our simulations - and thus need for starting with very high initial gas fractions - complicates a precise determination of the relative flux densities and duty cycles of the galaxy-pair and starburst phases. Regardless, it is clear that the galaxy-pair phase increases the SMG duty cycle significantly, alleviating some of the tension between the submm counts estimated from high-redshift major merger rates and short $(\sim 100 \mathrm{Myr})$ starburst duty cycles by Davé et al. (2010) and the observed submm counts. Inclusion of both SMG populations is crucial to match the observed SMG number counts without resorting to a top-heavy initial mass function (Hayward et al. 2011, C. Hayward et al. 2011, in preparation).

\subsection{Implications for IR SED fitting}

One reason the single-temperature, optically thin modified blackbody fails is that the effective optical depth of our simulated SMGs can be greater than 1 out to rest-frame $\sim 200 \mu \mathrm{m}$. This is consistent with the effective optical depths derived by Lupu et al. (2010) and Conley et al. (2011) when they fit a general modified blackbody (i.e., not assuming optical thinness) to the IR SEDs of their SMGs. By assuming optical thinness and only fitting longward of the FIR peak, one overestimates the luminosity density at wavelengths for which $\tau \gtrsim 1$ (see 3.2 .215 Consequently, the assumption of optical thinness yields a colder dust temperature (by as much as $\sim 20 \mathrm{~K}$ ) than if optical thinness is assumed.

In the pre-Herschel era, often flux densities in only a few FIR bands and at $1.4 \mathrm{GHz}$ were available for large samples of SMGs. As a result of the limited number of data points, models more complex than the optically thin modified blackbody (e.g., the full modified blackbody used here or models assuming a distribution of temperatures) could not provide a better description of the IR SEDs (e.g., Kovács et al. 2006). Recently, some authors

15 This may explain why Pope et al. (2006) found that the submm flux density tends to overpredict $L_{\mathrm{IR}}$, and the overprediction is worse for SMGs at low redshift where the SED is sampled further from the IR peak. have used models incorporating a distribution of temperatures (e.g., Kovács et al. 2010; Michałowski et al. 2010a), finding that such models better described the IR SEDs (Kovács et al. 2010). Now that Herschel PACS (Dannerbauer et al. 2010; Magnelli et al. 2010) and SPIRE (Chapman et al. 2010) have provided data over the entire FIR SED for large samples of SMGs it is possible to perform more sophisticated fitting for many SMGs. Given the physical inferences that are drawn from effective dust temperatures obtained via FIR SED fitting, it is important to have as robust a method as possible and to take full advantage of the available data. We will present such a method in future work.

\subsection{Limitations of our model}

At this point we find it instructive to define the limitations of this work so that the results can be placed in an appropriate context and future experiments can be designed for maximal impact. One of the primary limitations, both for the radiative transfer and the hydrodynamics, is the treatment of the sub-resolution interstellar medium, especially - because we focus upon the submm flux - the structure, distribution, and composition of the dust which dominates emission at submm wavelengths. In fact, the differences between the model employed in this work and that we used in N10 were motivated by a desire for a simpler treatment of the sub-resolution ISM (see 2.2 .1 ). In N10, stars with age $\leq 10$ Myr dominated the submm flux, so the submm flux was closely tied to the SFR (see Fig. 1 of N10). Our simplified assumptions (no sub-resolution PDR model, uniform ISM density on scales below the SPH smoothing length) result in submm flux that is tied more directly to the bolometric luminosity than the instantaneous star formation rate because stars with age $>10$ Myr contribute significantly to the bolometric luminosity and, because they are still deeply embedded in dust, the submm flux.

While our model has the advantages of simplicity and strict physical consistency (because the obscuration originates purely from the hydrodynamic simulations rather than from sub-resolution PDRs), we still must make an assumption about the sub-resolution structure of the ISM. By assuming uniform density on scales below the smoothing length we only include clumpiness that arises from the hydrodynamic simulations, so this assumption may be considered conservative. The assumption is also simplistic, of course, because the real ISM has significant structure on scales $\lesssim 100$ pc. However, proper treatment of radiative transfer through a clumpy medium is a significant area of research in and of itself (e.g., Hobson \& Padman 1993; Witt \& Gordon 1996; Városi \& Dwek 1999) and thus beyond the scope of this work. A study of the effects of sub-resolution dust clumpiness on galaxy SEDs and efforts to devise a better treatment of sub-resolution dust clumpiness in SUNRISE are underway.

We also caution that modifications to the simple star formation prescription and ISM treatment in the hydrodynamical simulations themselves could change the amplitude and duration of starbursts (Cox et al. 2006b; Springel et al. 2005). However, while changing the SF prescription or ISM treatment could change the relative contribution of quiescent and starburst star formation modes to the star formation history of a given 
merger, this alone should not change the differences between quiescent star formation and starbursts which lead to the significantly different relationships between SFR and submm flux. Changing the SF or ISM prescription could substantially alter the spatial distribution of dust and stars and thereby modify the detailed relations between $\mathrm{SFR} / L_{\mathrm{bol}}, M_{d}$, and $S_{850}$. However, because geometry is relatively unimportant in setting the relations, this uncertainty should have a relatively limited influence on our results. Furthermore, changing the feedback implementation can alter the evolution of the IR SEDs (Chakrabarti et al. 2007). Ongoing and future studies, with much higher resolution and more advanced tracking of the clumpy ISM (e.g., Hopkins et al. 2011), will improve the predictive power of our models.

Furthermore, we stress that the simulations presented here are not cosmological. We adopt this approach because it enables us to achieve the high resolution necessary to perform radiative transfer in order to accurately calculate the submm flux density; to survey the parameter space of progenitor masses, mass ratios, and orbits; and to avoid uncertainties in modeling realistic galaxy populations in a cosmological environment. The primary drawback of this approach for our present purposes is the lack of gas accretion, which cosmological hydrodynamic simulations show is a significant driver of star formation for the high-redshift, massive galaxies with which we are concerned (Kereš et al. 2005, 2009; Dekel et al. 2009). Gas accretion can continually replenish the gas in the galaxy, maintaining relatively high gas fractions and relatively constant star formation histories (e.g., Davé et al. 2010).

Inclusion of cosmological gas accretion would alter the time evolution presented in Figures 1, but it would not significantly alter the differences between the quiescent and merger-induced burst modes of star formation. This is because mergers would still superimpose a strong burst of star formation and sharp decrease in gas mass over the baseline evolution. Furthermore, unless smooth accretion significantly affects the geometry of stars and dust in the simulated galaxies, it will not have a significant effect on the relationship between submm flux, $L_{\text {bol }}$, and dust mass (the relationship between submm flux, SFR, and dust mass may be more affected because the relation between SFR and $L_{\text {bol }}$ may be changed significantly). Thus inclusion of cosmological gas accretion should not qualitatively alter our results.

\subsection{Connections to previous work}

In previous work, we developed a model relating the evolution of galaxies, starbursts, and quasars (Hopkins et al. 2006a, b, 2008a, 2009d; Somerville et al. 2008). A principal conclusion from these analyses is that while starbursts driven by gas-rich mergers can account for many instances of unusual activity in galaxies, they provide only a minor contribution to the star formation history of the Universe (Hopkins et al. 2006c). Indeed, Hopkins et al. (2010) emphasize that much of the star formation during galaxy interactions occurs in the "quiescent" mode and should not be counted as part of a merger-induced starburst. This is supported by the decomposition of the light profiles of nearby ongoing mergers (Hopkins et al. 2008b) and local cusp (Hopkins et al. 2009a) and core (Hopkins et al. 2009c) ellipticals. All of these objects exhibit evidence of "excess" central light (Rothberg \& Joseph 2004; Kormendy et al. 2009), indicative of relic starbursts (Mihos \& Hernquist 1994, 1996) caused by mergerdriven inflows of gas (Barnes \& Hernquist 1991, 1996). The integrated mass in these components agrees well with estimates of the cosmic history of merger-induced starbursts (Hopkins \& Hernquist 2010).

The results presented herein extend these conclusions to high-redshift phenomena. Critically, we find that "quiescent" star formation during galaxy interactions, i.e., star formation which occurs during the infall/pair stage, is a key element in understanding the brightest submm sources, especially their number counts and duty cycles, and connecting them to other highredshift populations including quasars (Hopkins et al. 2008a; Naravanan et al. 2008b) and compact spheroidal galaxies (Wuyts et al. 2009, 2010). Just as nearby LIRGs represent a heterogeneous collection of merging and isolated systems, it is natural to suggest that the population of high-redshift submm galaxies is heterogeneous, as we have argued here.

\section{CONCLUSIONS}

We have combined high-resolution 3-D hydrodynamic simulations of high-redshift isolated and merging disk galaxies and 3-D Monte Carlo dust radiative transfer calculations to study the submillimeter galaxy selection, focusing on the relationships among submm flux density, star formation rate, bolometric luminosity, and dust mass. Our main conclusions are the following:

1. The relationship between SFR and submm flux density differs significantly for quiescent and starburst star formation modes. Starbursts produce significantly less submm flux density for a given SFR, and the scaling between submm flux density and SFR is significantly weaker for bursts than for quiescent star formation. Bursts are a very inefficient way to boost submm flux density (e.g., a starburst that increases SFR by $\gtrsim 16 \mathrm{x}$ increases submm flux density by $\lesssim 2 \mathrm{x}$ ). Another consequence is that the galaxies with highest submm flux density are not necessarily those with highest SFR or bolometric or infrared luminosity.

2. The submm flux density of our simulations can be parameterized as a power law in SFR and dust mass $\left(L_{\mathrm{bol}}\right.$ and dust mass) to within $\sim 0.1(0.15)$ dex. The scaling derived from the commonly used optically thin modified blackbody model systematically overpredicts the submm flux density by $\gtrsim 2 \mathrm{x}$ because numerous assumptions of the model (optical thinness in the FIR, $\left.L_{\mathrm{IR}} \propto \mathrm{SFR}, L_{\mathrm{bol}} \approx L_{\mathrm{IR}}\right)$ do not hold. The fitting functions we provide (Equations (1) and 2) should be useful for calculating the flux density in semi-analytical models and cosmological simulations when full radiative transfer cannot be performed and for interpreting observations.

3. Mergers create SMGs through another mechanism besides the strong starburst induced at coalescence - they cause the two infalling disks to be observed as one submm source because both 
disks will be within the large $(\sim 15 "$, or $130 \mathrm{kpc}$ at $z=2$ ) beam of the single-dish submm telescopes used to identify SMGs during much of the infall stage. For major mergers, this effect boosts the submm flux density by $2 \mathrm{x}$. To achieve the same boost in submm flux density one would have to boost the SFR of a quiescent disk by $\sim 6 \mathrm{x}$ or induce a starburst that boosts the SFR by $\gtrsim 16 x$. This implies that the SMG population is heterogeneous: it is composed of both late-stage major mergers and two (or more) infalling disks observed as a single submm source ("galaxy-pair SMGs"). The largest quiescently star-forming galaxies may also contribute. Thus, unlike local ULIRGs, SMGs are a mix of quiescent and starburst sources.

4. SMGs must be very massive: to reach $S_{850} \gtrsim 3$ mJy, stellar mass of at least $6 \times 10^{10} M_{\odot}$ is required, and typical values are higher.

5. The submm duty cycles of our simulated galaxies are a factor of a few longer than what one would expect if all SMGs were merger-driven bursts because the relatively gentle decline in $\mathrm{SFR}, L_{\mathrm{bol}}$, and dust mass during the galaxy-pair phase results in a longer duty cycle for the galaxy-pair phase than for the starburst. The duty cycle of the latter is limited because the peak in luminosity is narrow and the dust temperature increases sharply during the burst.

6. Fitting the SEDs of SMGs with an optically thin modified blackbody tends to yield significantly lower dust temperatures than when the full opacity term is used because the effective optical depths can be $\sim 1$ out to rest-frame $\sim 200 \mu \mathrm{m}$, both for our simulated SMGs and observed SMGs. Therefore, one should be cautious when interpreting effective dust temperatures derived via fitting an optically thin modified blackbody to the FIR SED, especially when comparing SMGs to galaxies for which optical thinness in the IR may be a reasonable approximation.

Future work will include predictions of submm number counts from our model, an investigation of the observational signatures and physical implications of the proposed SMG bimodality, and an improved method for fitting IR SEDs of galaxies.

CCH thanks Scott Chapman, Emanuele Daddi, Helmut Dannerbauer, Phil Hopkins, Brandon Kelly, Chris McKee, Michał Michałowski, Diego Munoz, Alex Pope, Barry Rothberg, Greg Snyder, and Josh Younger for useful discussion and Jeff Pettibone for hospitality which facilitated the writing of this paper. We thank Volker Springel for providing the non-public version of GADGET-2 used for this work and Brant Robertson for use of his code to generate initial disk galaxies scaled to high redshift. DK is supported by NASA through Hubble Fellowship grant HST-HF-51276.01-A. PJ acknowledges support by a grant from the W. M. Keck Foundation. DN acknowledges support from a National Science Foundation Grant (AST-1009452). The simulations in this paper were performed on the Odyssey cluster supported by the FAS Research Computing Group at Harvard University.

\section{REFERENCES}

Alexander, D. M., Bauer, F. E., Chapman, S. C., Smail, I., Blain, A. W., Brandt, W. N., \& Ivison, R. J. 2005a, ApJ, 632, 736

Alexander, D. M., Smail, I., Bauer, F. E., Chapman, S. C., Blain A. W., Brandt, W. N., \& Ivison, R. J. 2005b, Nature, 434, 738 Alexander, D. M., et al. 2008, AJ, 135, 1968

Barger, A. J., Cowie, L. L., Sanders, D. B., Fulton, E., Taniguchi, Y., Sato, Y., Kawara, K., \& Okuda, H. 1998, Nature, 394, 248 Barnes, J., \& Hernquist, L. 1991, ApJ, 370, L65

-. 1996, ApJ, 471, 115

Barnes, J., \& Hut, P. 1986, Nature, 324, 446

Baugh, C. M., Lacey, C. G., Frenk, C. S., Granato, G. L., Silva, L., Bressan, A., Benson, A. J., \& Cole, S. 2005, MNRAS, 356, 1191

Biggs, A. D., \& Ivison, R. J. 2008, MNRAS, 385, 893

Blain, A. W., Smail, I., Ivison, R. J., Kneib, J.-P., \& Frayer,

D. T. 2002, Phys. Rep., 369, 111

Bothwell, M. S., et al. 2010, MNRAS, 405, 219

Bouché, N., et al. 2007, ApJ, 671, 303

Brodwin, M. et al. 2008, ApJ, 687, L65

Bush, S. J., Cox, T. J., Hayward, C. C., Thilker, D., Hernquist, L., \& Besla, G. 2010, ApJ, 713, 780

Bussmann, R. S., et al. 2009a, ApJ, 693, 750

-. 2009b, ApJ, 705, 184

Calzetti, D. 1997, AJ, 113, 162

Calzetti, D., Armus, L., Bohlin, R. C., Kinney, A. L., Koornneef, J., \& Storchi-Bergmann, T. 2000, ApJ, 533, 682

Calzetti, D., Kinney, A. L., \& Storchi-Bergmann, T. 1994, ApJ, 429,582

Capak, P., et al. 2008, ApJ, 681, L53

Carilli, C. L., et al. 2010, ApJ, 714, 1407

Casey, C. M., Chapman, S. C., Smail, I., Alaghband-Zadeh, S., Bothwell, M. S., \& Swinbank, A. M. 2010, arXiv:1009.5709

Casey, C. M., et al. 2009, MNRAS, 399, 121

Chakrabarti, S., Cox, T. J., Hernquist, L., Hopkins, P. F.,

Robertson, B., \& Matteo, T. D. 2007, ApJ, 658, 840

Chakrabarti, S., Fenner, Y., Cox, T. J., Hernquist, L., \& Whitney, B. A. 2008, ApJ, 688, 972

Chakrabarti, S., \& McKee, C. F. 2005, ApJ, 631, 792

-. 2008, ApJ, 683, 693

Chakrabarti, S., \& Whitney, B. A. 2009, ApJ, 690, 1432

Chapman, S. C., Blain, A. W., Smail, I., \& Ivison, R. J. 2005, ApJ, 622, 772

Chapman, S. C., Smail, I., Blain, A. W., \& Ivison, R. J. 2004, ApJ, 614, 671

Chapman, S. C., Windhorst, R., Odewahn, S., Yan, H., \& Conselice, C. 2003, ApJ, 599, 92

Chapman, S. C., et al. 2000, MNRAS, 319, 318

Chapman, S. C., et al. 2010, MNRAS, 409, L13

Chary, R., \& Elbaz, D. 2001, ApJ, 556, 562

Clements, D. L. Dunne, L. \& Eales, S. 2010, MNRAS, 403, 274

Conley, A., et al. 2011, ApJ, 732, L35

Coppin, K., et al. 2008, MNRAS, 384, 1597

Cox, T. J., Dutta, S. N., Matteo, T. D., Hernquist, L., Hopkins,

P. F., Robertson, B., \& Springel, V. 2006a, ApJ, 650, 791

Cox, T. J., Jonsson, P., Primack, J. R., \& Somerville, R. S. 2006b, MNRAS, 373, 1013

Cox, T. J., Jonsson, P., Somerville, R. S., Primack, J. R., \& Dekel, A. 2008, MNRAS, 384, 386

Daddi, E., Dannerbauer, H., Krips, M., Walter, F., Dickinson, M. Elbaz, D., \& Morrison, G. E. 2009a, ApJ, 695, L176

Daddi, E., et al. 2005, ApJ, 631, L13

—. 2007, ApJ, 670, 156

-. 2009b, ApJ, 694, 1517

- 2010, ApJ, 713, 686

Dale, D. A., \& Helou, G. 2002, ApJ, 576, 159

Dale, D. A., Helou, G., Contursi, A., Silbermann, N. A., \& Kolhatkar, S. 2001, ApJ, 549, 215

Dale, D. A., et al. 2007, ApJ, 655, 863

Dannerbauer, H., Daddi, E., Riechers, D. A., Walter, F., Carilli, C. L., Dickinson, M., Elbaz, D., \& Morrison, G. E. 2009, ApJ 698, L178

Dannerbauer, H., Lehnert, M. D., Lutz, D., Tacconi, L., Bertoldi, F., Carilli, C., Genzel, R., \& Menten, K. 2002, ApJ, 573, 473

Dannerbauer, H., et al. 2010, ApJ, 720, L144

Davé, R., Finlator, K., Oppenheimer, B. D., Fardal, M., Katz, N., Kereš, D., \& Weinberg, D. H. 2010, MNRAS, 404, 1355

Dekel, A., et al. 2009, Nature, 457, 451

Downes, D., \& Solomon, P. M. 1998, ApJ, 507, 615

Draine, B. T., \& Li, A. 2007, ApJ, 657, 810

Dunne, L., \& Eales, S. A. 2001, MNRAS, 327, 697 
Dunne, L., Eales, S. A., \& Edmunds, M. G. 2003, MNRAS, 341, 589

Dwek, E. 1998, ApJ, 501, 643

Eales, S., Lilly, S., Gear, W., Dunne, L., Bond, J. R., Hammer, F., Fèvre, O. L., \& Crampton, D. 1999, ApJ, 515, 518

Edmunds, M. G., \& Eales, S. A. 1998, MNRAS, 299, L29

Engel, H., et al. 2010, arXiv:1009.2495

Erb, D. K., Steidel, C. C., Shapley, A. E., Pettini, M., Reddy, N. A., \& Adelberger, K. L. 2006, ApJ, 646, 107

Fontanot, F., \& Monaco, P. 2010, MNRAS, 405, 705

Fontanot, F., Monaco, P., Silva, L., \& Grazian, A. 2007, MNRAS, 382,903

Gingold, R. A., \& Monaghan, J. J. 1977, MNRAS, 181, 375

González, J. E., Lacey, C. G., Baugh, C. M., \& Frenk, C. S. 2011, MNRAS, 413, 749

Granato, G. L., De Zotti, G., Silva, L., Bressan, A., \& Danese, L. 2004, ApJ, 600, 580

Greve, T. R., Pope, A., Scott, D., Ivison, R. J., Borys, C., Conselice, C. J., \& Bertoldi, F. 2008, MNRAS, 389, 1489

Greve, T. R., et al. 2005, MNRAS, 359, 1165

Groves, B., Dopita, M. A., Sutherland, R. S., Kewley, L. J. Fischera, J., Leitherer, C., Brandl, B., \& van Breugel, W. 2008 ApJS, 176,438

Hainline, L. J., Blain, A. W., Smail, I., Alexander, D. M., Armus, L., Chapman, S. C., \& Ivison, R. J. 2010, arXiv:1006.0238

Hayward, C. C., Narayanan, D., Jonsson, P., Cox, T. J., Kereš, D., Hopkins, P. F., \& Hernquist, L. 2011, in Astronomical Society of the Pacific Conference Series, Vol. 440, Astronomical Society of the Pacific Conference Series, ed. M. Treyer, T. Wyder, J. Neill, M. Seibert, \& J. Lee, 369-+

Hernquist, L. 1990, ApJ, 356, 359

Hernquist, L., \& Katz, N. 1989, ApJS, 70, 419

Hobson, M. P., \& Padman, R. 1993, MNRAS, 264, L161

Holland, W. S., et al. 1999, MNRAS, 303, 659

Hopkins, P. F., Cox, T. J., Dutta, S. N., Hernquist, L., Kormendy, J., \& Lauer, T. R. 2009a, ApJS, 181, 135

Hopkins, P. F., Cox, T. J., Kereš, D., \& Hernquist, L. 2008a, ApJS, 175, 390

Hopkins, P. F., Cox, T. J., Younger, J. D., \& Hernquist, L. 2009b ApJ, 691, 1168

Hopkins, P. F., \& Hernquist, L. 2010, MNRAS, 402, 985

Hopkins, P. F., Hernquist, L., Cox, T. J., Di Matteo, T., Robertson, B., \& Springel, V. 2006a, ApJS, 163, 1

Hopkins, P. F., Hernquist, L., Cox, T. J., Dutta, S. N., \& Rothberg, B. 2008b, ApJ, 679, 156

Hopkins, P. F., Hernquist, L., Cox, T. J., \& Kereš, D. 2008c, ApJS, 175,356

Hopkins, P. F., Hernquist, L., Cox, T. J., Robertson, B., \& Springel, V. 2006b, ApJS, 163, 50

Hopkins, P. F., Lauer, T. R., Cox, T. J., Hernquist, L., \& Kormendy, J. 2009c, ApJS, 181, 486

Hopkins, P. F., Quataert, E., \& Murray, N. 2011, arXiv:1101.4940

Hopkins, P. F., Richards, G. T., \& Hernquist, L. 2007, ApJ, 654, 731

Hopkins, P. F., Somerville, R. S., Hernquist, L., Cox, T. J., Robertson, B., \& Li, Y. 2006c, ApJ, 652, 864

Hopkins, P. F., Younger, J. D., Hayward, C. C., Narayanan, D., \& Hernquist, L. 2010, MNRAS, 402, 1693

Hopkins, P. F., et al. 2009d, MNRAS, 397, 802

Hughes, D. H., et al. 1998, Nature, 394, 241

Hwang, H. S., et al. 2010, MNRAS, 409, 75

Iono, D., et al. 2009, ApJ, 695, 1537

Ivison, R. J., Smail, I., Papadopoulos, P. P., Wold, I., Richard, J., Swinbank, A. M., Kneib, J., \& Owen, F. N. 2010, MNRAS, 404, 198

Ivison, R. J., et al. 2002, MNRAS, 337, 1

-. 2007, MNRAS, 380, 199

James, A., Dunne, L., Eales, S., \& Edmunds, M. G. 2002 MNRAS, 335,753

Jonsson, P. 2006, MNRAS, 372, 2

Jonsson, P., Cox, T. J., Primack, J. R., \& Somerville, R. S. 2006 ApJ, 637, 255

Jonsson, P., Groves, B. A., \& Cox, T. J. 2010, MNRAS, 403, 17

Jonsson, P., \& Primack, J. R. 2010, NewA, 15, 509

Juvela, M. 2005, A\&A, 440, 531

Katz, N., Weinberg, D. H., \& Hernquist, L. 1996, ApJS, 105, 19

Kennicutt, R. C. 1998a, ApJ, 498, 541

- 1998b, ARA\&A, 36, 189

Kennicutt, Jr., R. C., et al. 2003, PASP, 115, 928

Kereš, D., Katz, N., Fardal, M., Davé, R., \& Weinberg, D. H. 2009, MNRAS, 395, 160

Kereš, D., Katz, N., Weinberg, D. H., \& Davé, R. 2005, MNRAS, 363,2

Knudsen, K. K., Kneib, J.-P., Richard, J., Petitpas, G., \& Egami, E. 2010, ApJ, 709, 210

Kormendy, J., Fisher, D. B., Cornell, M. E., \& Bender, R. 2009, ApJS, 182, 216
Kovács, A., Chapman, S. C., Dowell, C. D., Blain, A. W., Ivison,

R. J., Smail, I., \& Phillips, T. G. 2006, ApJ, 650, 592

Kovács, A., et al. 2010, ApJ, 717, 29

Kroupa, P. 2001, MNRAS, 322, 231

Krumholz, M. R., \& Thompson, T. A. 2007, ApJ, 669, 289

Leitherer, C., et al. 1999, ApJS, 123, 3

Lisenfeld, U., Isaak, K. G., \& Hills, R. 2000, MNRAS, 312, 433

Lo Faro, B., Monaco, P., Vanzella, E., Fontanot, F., Silva, L., \& Cristiani, S. 2009, MNRAS, 399, 827

Lonsdale, C. J., Farrah, D., \& Smith, H. E. 2006, Ultraluminous

Infrared Galaxies, ed. Mason, J. W. (Springer Verlag), 285

Lucy, L. B. 1977, AJ, 82, 1013

Lupu, R. E., et al. 2010, arXiv:1009.5983

Magdis, G. E., et al. 2010, MNRAS, 409, 22

Magnelli, B., et al. 2010, A\&A, 518, L28

Matteo, T. D., Springel, V., \& Hernquist, L. 2005, Nature, 433, 604

Menéndez-Delmestre, K., et al. 2007, ApJ, 655, L65

-. 2009, ApJ, 699, 667

Michałowski, M., Hjorth, J., \& Watson, D. 2010a, A\&A, 514, A67

Michałowski, M. J., Watson, D., \& Hjorth, J. 2010b, ApJ, 712, 942

Mihos, J. C., \& Hernquist, L. 1994, ApJ, 437, L47

-. 1996, ApJ, 464, 641

Narayanan, D., Cox, T. J., Hayward, C. C., \& Hernquist, L. 2011, MNRAS, 412, 287

Narayanan, D., Cox, T. J., Hayward, C. C., Younger, J. D., \&

Hernquist, L. 2009, MNRAS, 400, 1919

Narayanan, D., Cox, T. J., Shirley, Y., Davé, R., Hernquist, L., \& Walker, C. K. 2008a, ApJ, 684, 996

Narayanan, D., Hayward, C. C., Cox, T. J., Hernquist, L., Jonsson, P., Younger, J. D., \& Groves, B. 2010a, MNRẢS, 401, $1613(\mathrm{~N} 10)$

Narayanan, D., et al. 2008b, ApJS, 174, 13

- 2010b, MNRAS, 407, 1701

Neri, R., et al. 2003, ApJ, 597, L113

Noeske, K. G., et al. 2007a, ApJ, 660, L47

-. 2007b, ApJ, 660, L43

Papadopoulos, P. P., van der Werf, P., Isaak, K., \& Xilouris, E. M. 2010, ApJ, 715, 775

Peacock, J. A., et al. 2000, MNRAS, 318, 535

Pope, A., et al. 2006, MNRAS, 370

-. 2008, ApJ, 675, 1171

Rangwala, N., et al. 2011, arXiv:1106.5054

Rex, M., et al. 2010, A\&A, 518, L13

Ricciardelli, E., Trujillo, I., Buitrago, F., \& Conselice, C. J. 2010, MNRAS, 406, 230

Robertson, B., Bullock, J. S., Cox, T. J., Di Matteo, T. Hernquist, L., Springel, V., \& Yoshida, N. 2006a, ApJ, 645, 986

Robertson, B., Hernquist, L., Cox, T. J., Matteo, T. D., Hopkins, P. F., Martini, P., \& Springel, V. 2006b, ApJ, 641, 90

Robertson, B. E., \& Bullock, J. S. 2008, ApJ, 685, L27

Rothberg, B., \& Fischer, J. 2010, ApJ, 712, 318

Rothberg, B., \& Joseph, R. D. 2004, AJ, 128, 2098

Sakamoto, K., Scoville, N. Z., Yun, M. S., Crosas, M., Genzel, R., \& Tacconi, L. J. 1999, ApJ, 514, 68

Sakamoto, K., et al. 2008, ApJ, 684, 957

Sanders, D. B., \& Mirabel, I. F. 1996, ARA\&A, 34, 749

Schinnerer, E., et al. 2008, ApJ, 689, L5

Scott, S. E., et al. 2002, MNRAS, 331, 817

Scoville, N. Z., Sargent, A. I., Sanders, D. B., \& Soifer, B. T. 1991, ApJ, 366, L5

Shetty, R., Kauffmann, J., Schnee, S., \& Goodman, A. A. 2009a, ApJ, 696, 676

Shetty, R., Kauffmann, J., Schnee, S., Goodman, A. A., \&

Ercolano, B. 2009b, ApJ, 696, 2234

Smail, I., Chapman, S. C., Blain, A. W., \& Ivison, R. J. 2004, ApJ, 616, 71

Smail, I. Ivison, R. J., \& Blain, A. W. 1997, ApJ, 490, L5

Snyder, G. F., Cox, T. J., Hayward, C. C., Hernquist, L., \& Jonsson, P. 2011, ArXiv e-prints, arXiv:1102.3689

Somerville, R. S., Hopkins, P. F., Cox, T. J., Robertson, B. E., \&

Hernquist, L. 2008, MNRAS, 391, 481

Springel, V. 2005, MNRAS, 364, 1105

Springel, V., \& Hernquist, L. 2002, MNRAS, 333, 649

-. 2003, MNRAS, 339, 289

Springel, V., Matteo, T. D., \& Hernquist, L. 2005, MNRAS, 361, 776

Springel, V., Yoshida, N., \& White, S. D. M. 2001, NewA, 6, 79

Swinbank, A. M., Smail, I., Chapman, S. C., Blain, A. W., Ivison

R. J., \& Keel, W. C. 2004, ApJ, 617, 64

Swinbank, A. M., et al. 2008, MNRAS, 391, 420

Tacconi, L. J., et al. 2006, ApJ, 640, 228

-. 2008, ApJ, 680, 246

-. 2010, Nature, 463, 781 
Targett, T. A., Dunlop, J. S., McLure, R. J., Best, P. N., Cirasuolo, M., \& Almaini, O. 2011, MNRAS, in press, arXiv: 1005.5176

Valiante, E., et al. 2007, ApJ, 660, 1060

van Kampen, E., et al. 2005, MNRAS, 359, 469

Városi, F., \& Dwek, E. 1999, ApJ, 523, 265

Wang, W.-H., Cowie, L. L., Barger, A. J., \& Williams, J. P. 2011, ApJ, 726, L18+

Webb, T. M., et al. 2003, ApJ, 582, 6

Weingartner, J. C., \& Draine, B. T. 2001, ApJ, 548, 296

Wilson, G. W., et al. 2008, MNRAS, 386, 807

Witt, A. N., \& Gordon, K. D. 1996, ApJ, 463, 681

Wright, E. L. 2006, PASP, 118, 1711
Wuyts, S., Cox, T. J., Hayward, C. C., Franx, M., Hernquist, L., Hopkins, P. F., Jonsson, P., \& van Dokkum, P. G. 2010, ApJ, 722,1666

Wuyts, S., et al. 2009, ApJ, 700, 799

Younger, J. D., Hayward, C. C., Narayanan, D., Cox, T. J.,

Hernquist, L., \& Jonsson, P. 2009a, MNRAS, 396, L66

Younger, J. D., et al. 2007, ApJ, 671, 1531

-. 2008, ApJ, 688, 59

-. 2009b, MNRAS, 394, 1685

-. 2009c, ApJ, 704, 803

-. 2010, MNRAS, 407, 1268

\section{APPENDIX}

\section{DERIVATION OF THE RELATIONS GIVEN IN $\$ 3.2 .1$}

Here we derive the relations for submm flux density as a function of dust bolometric luminosity $L_{d}$ and dust mass $M_{d}$ (Equation 4) and SFR and $M_{d}$ (Equation 3) for an optically thin modified blackbody. One can model galaxy SEDs with more complex models (e.g., Dale et al. 2001; Dale \& Helou 2002; Chakrabarti \& McKee 2005, 2008; Kovács et al. 2010), but for the sake of simplicity and because the optically thin modified blackbody is commonly used for SED fitting we only consider an optically thin modified blackbody here. Consider a mass $M_{d}$ of dust with temperature $T_{d}$. Assuming the dust is optically thin at rest-frame frequency $\nu_{r}$, the luminosity density emitted by the dust at that frequency is

$$
L_{\nu_{r}}=4 \pi \kappa_{\nu_{r}} M_{d} B_{\nu_{r}}\left(T_{d}\right),
$$

where $\kappa_{\nu_{r}}$ is the dust opacity $\left(\mathrm{m}^{2} \mathrm{~kg}^{-1}\right)$ at rest-frame frequency $\nu_{r}$ and $B_{\nu_{r}}\left(T_{d}\right)$ is the Planck function. We assume a power-law opacity in the IR,

$$
\kappa_{\nu_{r}}=\kappa_{0}\left(\frac{\nu_{r}}{\nu_{0}}\right)^{\beta},
$$

where $\kappa_{0}$ is the opacity at frequency $\nu_{0}$. Integrating Equation (A1) over $\nu$ gives the total dust luminosity,

$$
L_{d}=\Gamma(4+\beta) \zeta(4+\beta) \frac{8 \pi h}{c^{2}}\left(\frac{k T_{d}}{h}\right)^{4} M_{d} \kappa_{0}\left(\frac{k T_{d}}{h \nu_{0}}\right)^{\beta},
$$

where $\Gamma$ and $\zeta$ are Riemann functions, $h$ is the Planck constant, $c$ is the speed of light, and $k$ is the Boltzmann constant.

Solving for the effective dust temperature yields

$$
T_{d}=\frac{h}{k}\left[\frac{L_{d} c^{2} \nu_{0}^{\beta}}{\Gamma(4+\beta) \zeta(4+\beta) 8 \pi \kappa_{0} h M_{d}}\right]^{1 /(4+\beta)} .
$$

If we place the mass of dust at redshift $z$, the flux density at observed-frame frequency $\nu_{o}$ is

$$
\begin{aligned}
S_{\nu_{o}}\left(T_{d}\right) & =(1+z) \frac{L_{\nu_{r}}}{4 \pi D_{L}^{2}} \\
& =(1+z) \frac{4 \pi \kappa_{\nu_{r}} M_{d} B_{\nu_{r}}\left(T_{d}\right)}{4 \pi D_{L}^{2}} \\
& =(1+z)^{\beta-3} \frac{\kappa_{0} M_{d}}{D_{A}^{2}}\left(\frac{\nu_{o}}{\nu_{0}}\right)^{\beta} B_{\nu_{o}(1+z)}\left(T_{d}\right),
\end{aligned}
$$

where we have related angular diameter distance $D_{A}$ and luminosity distance $D_{L}$ using $D_{L}=(1+z)^{2} D_{A}$. In the Rayleigh-Jeans limit, $B_{\nu}(T)=2 k \nu^{2} T / c^{2}$, so

$$
S_{\nu_{o}}\left(T_{d}\right)=(1+z)^{\beta-1} \frac{2 k \kappa_{0}}{c^{2} D_{A}^{2}}\left(\frac{\nu_{o}}{\nu_{0}}\right)^{\beta} \nu_{o}^{2} M_{d} T_{d} .
$$

By substituting $T_{d}$ from Equation (A4) into Equation (A8) we find

$$
S_{\nu_{o}}=\frac{2 h \kappa_{0}}{c^{2} D_{A}^{2}} \nu_{o}^{2}\left(\frac{\nu_{o}}{\nu_{0}}\right)^{\beta}\left(\frac{\nu_{0}^{\beta} c^{2}}{\Gamma(4+\beta) \zeta(4+\beta) 8 \pi \kappa_{0} h}\right)^{1 /(4+\beta)}(1+z)^{\beta-1} L_{d}^{1 /(4+\beta)} M_{d}^{(3+\beta) /(4+\beta)}
$$

For the Weingartner \& Draine (2001) $R_{V}=3.1$ Milky Way dust model, which we use in our RT calculations, $\beta \approx 2$ and the $850 \mu \mathrm{m}$ opacity is $\kappa_{850}=0.050 \mathrm{~m}^{2} \mathrm{~kg}^{-1}$, consistent with the value James et al. (2002) derived from submm 
observations of local galaxies, $0.07 \pm 0.02 \mathrm{~m}^{2} \mathrm{~kg}^{-1}$, and with the results of Dunne et al. (2003). Thus the observed $850 \mu \mathrm{m}$ flux density is

$$
S_{850}=1.5 \mathrm{mJy}(1+z)\left(\frac{D_{A}}{1 \mathrm{Gpc}}\right)^{-2}\left(\frac{L_{d}}{10^{12} L_{\odot}}\right)^{1 / 6}\left(\frac{M_{d}}{10^{8} M_{\odot}}\right)^{5 / 6} .
$$

The Kennicutt (1998b) SFR- $L_{\mathrm{IR}}$ calibration converted to a Kroupa (2001) initial mass function is

$$
L_{\mathrm{IR}} \approx L_{\mathrm{bol}} \approx 9 \times 10^{9} L_{\odot}\left(\mathrm{SFR} / M_{\odot} \mathrm{yr}^{-1}\right)
$$

This conversion assumes all starlight is absorbed by dust and the contribution from AGN and old stars is negligible; as discussed above, these assumptions are all violated at some level. (If these assumption were true, the power-law indices in Equations (1) and (2) would be identical.) However, since the above calibration is ubiquitously applied, we will give the relation that results when we use it; the relation is

$$
S_{850}=1.5 \text { mJy }(1+z)\left(\frac{D_{A}}{1 \mathrm{Gpc}}\right)^{-2}\left(\frac{\mathrm{SFR}}{100 M_{\odot} \mathrm{yr}^{-1}}\right)^{1 / 6}\left(\frac{M_{d}}{10^{8} M_{\odot}}\right)^{5 / 6} .
$$

Assuming $\Omega_{m}=0.27, \Omega_{\Lambda}=0.73$, and $h=0.7$, the angular diameter distance at $z=2$ is $1.77 \mathrm{Gpc}$ (Wright 2006 ). Thus for $z=2$ we recover Equation (4),

$$
S_{850}=1.4 \mathrm{mJy}\left(\frac{L_{d}}{10^{12} L_{\odot}}\right)^{1 / 6}\left(\frac{M_{d}}{10^{8} M_{\odot}}\right)^{5 / 6} .
$$

This should be compared to Equation (2). In terms of SFR, we get Equation (3),

$$
S_{850}=1.4 \mathrm{mJy}\left(\frac{\mathrm{SFR}}{100 M_{\odot} \mathrm{yr}^{-1}}\right)^{1 / 6}\left(\frac{M_{d}}{10^{8} M_{\odot}}\right)^{5 / 6} .
$$

This should be compared to Equation (11).

Even if the underlying power-law index of the dust opacity curve is $\beta=2$, for a distribution of dust temperatures a single-temperature modified blackbody with $\beta=1.5$ may better fit the SED (Dunne \& Eales 2001; Chakrabarti \& McKee 2008). Note also that the fitted dust temperature and $\beta$ are degenerate, and the fitted values can depend sensitively on both noise in the data and temperature variations along the line-of-sight (Shettv et al. $2009 \mathrm{a}, \mathrm{b})$. Since $\beta=1.5$ is often assumed when fitting SEDs and determining dust masses of SMGs (e.g., Kovács et al. 2006, 2010; Coppin et al. 2008; Chapman et al. 2010), so we will provide the relations for $\beta=1.5$ also. They are:

$$
S_{850}=1.9 \text { mJy }(1+z)^{0.5}\left(\frac{D_{A}}{1 \mathrm{Gpc}}\right)^{-2}\left(\frac{L_{d}}{10^{12} L_{\odot}}\right)^{0.18}\left(\frac{M_{d}}{10^{8} M_{\odot}}\right)^{0.82},
$$

and

$$
S_{850}=1.9 \text { mJy }(1+z)^{0.5}\left(\frac{D_{A}}{1 \mathrm{Gpc}}\right)^{-2}\left(\frac{\mathrm{SFR}}{100 M_{\odot} \mathrm{yr}^{-1}}\right)^{0.18}\left(\frac{M_{d}}{10^{8} M_{\odot}}\right)^{0.82} .
$$

For $z=2$,

$$
S_{850}=1.0 \mathrm{mJy}\left(\frac{L_{d}}{10^{12} L_{\odot}}\right)^{0.18}\left(\frac{M_{d}}{10^{8} M_{\odot}}\right)^{0.82}
$$

and

$$
S_{850}=1.0 \mathrm{mJy}\left(\frac{\mathrm{SFR}}{100 M_{\odot} \mathrm{yr}^{-1}}\right)^{0.18}\left(\frac{M_{d}}{10^{8} M_{\odot}}\right)^{0.82} .
$$

The equations can be rescaled to different values of $\kappa_{0}$ using $S_{\nu_{o}} \propto \kappa_{0}^{(3+\beta) /(4+\beta)}$ and to different submm wavelengths using $S_{\nu_{o}} \propto \nu_{o}^{2+\beta}$ (see Equation A9). 